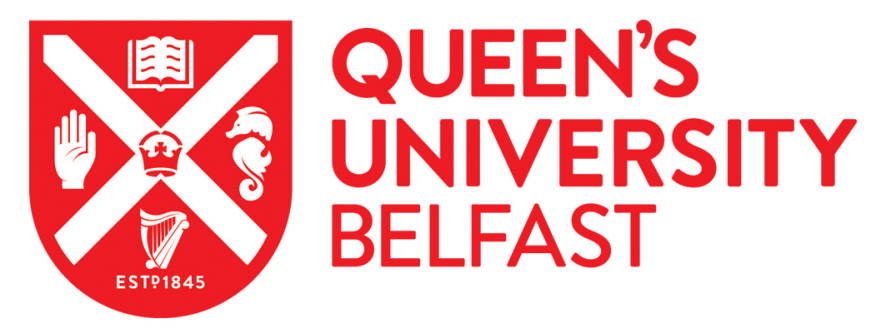

\title{
An overview of seismic hybrid testing of engineering structures
}

McCrum, D. P., \& Williams, M. S. (2016). An overview of seismic hybrid testing of engineering structures.

Engineering Structures, 118, 240-261. https://doi.org/10.1016/j.engstruct.2016.03.039

\author{
Published in: \\ Engineering Structures
}

Document Version:

Peer reviewed version

Queen's University Belfast - Research Portal:

Link to publication record in Queen's University Belfast Research Portal

Publisher rights

(c) 2016 Elsevier Ltd. This manuscript version is made available under the CC-BY-NC-ND 4.0 license http://creativecommons.org/licenses/by$\mathrm{nc}-\mathrm{nd} / 4.0 /$ which permits distribution and reproduction for non-commercial purposes, provided the author and source are cited.

\section{General rights}

Copyright for the publications made accessible via the Queen's University Belfast Research Portal is retained by the author(s) and / or other copyright owners and it is a condition of accessing these publications that users recognise and abide by the legal requirements associated with these rights.

Take down policy

The Research Portal is Queen's institutional repository that provides access to Queen's research output. Every effort has been made to ensure that content in the Research Portal does not infringe any person's rights, or applicable UK laws. If you discover content in the Research Portal that you believe breaches copyright or violates any law, please contact openaccess@qub.ac.uk. 
Title: An Overview of Seismic Hybrid Testing of Engineering Structures

Author's names: D. P. McCrum ${ }^{1} \&$ M. S. Williams ${ }^{2}$

Author's Affiliations: 1 - School of Planning, Architecture and Civil Engineering, Queen's University of Belfast, Belfast BT9 5AG, UK. 2 - Department of Engineering Science, University of Oxford, Parks Road, Oxford OX1 3PJ, UK.

Correspondence to: Daniel P. McCrum, School of Planning, Architecture and Civil Engineering, David Keir Building, Queen’s University Belfast, BT9 5AG, United Kingdom

E-mail: d.mccrum@qub.ac.uk

Abstract: An overview of research on the development of the hybrid test method is presented. The maturity of the hybrid test method is mapped in order to provide context to individual research in the overall development of the test method. In the pseudo dynamic (PsD) test method, the equations of motion are solved using a time stepping numerical integration technique with the inertia and damping being numerically modelled whilst restoring force is physically measured over an extended timescale. Developments in continuous PsD testing led to the real-time hybrid test method and geographically distributed hybrid tests. A key aspect to the efficiency of hybrid testing is the substructuring technique where the critical structural subassemblies that are fundamental to the overall response of the structure are physically tested whilst the remainder of the structure whose response can be more easily predicted is numerically modelled. Much of the early research focused on developing the accuracy and efficiency of the test method, whereas more recently the method has matured to a level where the test method is applied purely as a dynamic testing technique. Developments in numerical integration methods, substructuring, experimental error reduction, delay compensation and speed of testing have led to a test method now in use as full-scale real-time dynamic testing method that is reliable, accurate, efficient and cost effective.

Keywords: seismic, pseudo dynamic testing, real-time hybrid testing, numerical time integration, geographically distributed testing

\section{INTRODUCTION}

This paper details the development of the hybrid test method. The term hybrid test is used here to describe the family of coupled physical-computational simulations of seismic response, in which a part of the structural behaviour is tested physically while other terms are 
modelled numerically. This definition includes pseudo-dynamic (PsD) testing (with or without substructuring) and real-time hybrid tests (RTHT). This family of test methods was developed to answer the need for realistic seismic testing of engineering structures without the costs and scaling issues associated with shake table testing of complete structural models. Starting from PsD testing in the 1970s, hybrid tests have developed in diverse ways to meet different testing needs. PsD testing has become a well-established and valuable technique that can be applied to complex structural models. RTHT has to date been applied to relatively small and simple test specimens, but is now approaching a level of maturity where it can be more widely used with confidence.

As a result of years of development, the hybrid test method is now being applied purely as a reliable dynamic test method. The main motivations now for performing hybrid tests, similar to those of other seismic dynamic testing techniques, are: (i) real-time testing of the rate dependent behaviour of dampers and base isolators; (ii) increased interest in modelling building performance up to collapse, evaluating the response to the Maximum Considered Earthquake rather than just the Design Basis Earthquake; (iii) understanding uncertainty around structural performance at extreme deformation levels; and (iv) increased interest in non-structural elements under lower level earthquakes.

This paper is structured as follows; Section 2 will discuss the development of founding hybrid test methods to its current form as a RTHT, including a brief discussion of the motivation for such testing. Section 3 will detail the development of time integration techniques that improve stability and efficiency of the hybrid test method, particularly for a RTHT. In Section 4, stability and accuracy of the hybrid test method is discussed in terms of actuator delay compensation techniques. The developments in geographically distributed hybrid testing are detailed in Section 5. The hybrid test method has now reached a level of maturity that it has become a standard method of dynamic testing and in Section 6 applications of the PsD and RTHT methods are discussed.

\section{BACKGROUND OF HYBRID TESTING}

The dynamic response of structures to extreme events such as earthquake is often difficult to model numerically with complete accuracy, particularly at extreme deformations. The difficulties often arise in structures that may undergo high levels of material nonlinearity, including cyclic degradation and low-cycle fatigue. Some structural components exhibit rate dependency in their response, and many seismic mitigation devices (e.g. dampers, inerters, visco-elastic materials) are based on rate dependent properties. The main types of structural 
laboratory testing under dynamic loads reviewed by Williams \& Blakeborough, [1] are: (i) quasi-static testing (ii) shake table testing (iii) PsD testing and more recently, (iv) RTHT. Another specific test method worth discussing is the effective force test (EFT) method; this is conceptually different to the PsD test method, but similarly to the PsD method can be applied to any structural system that can be idealised with lumped masses.

Quasi-static testing, such as the static cyclic test method, is typically used to assess the onset and development of non-linearity in structural elements under reversed cyclic loads. This has provided the basis for codes and guidelines on element design. However, there remains a need to understand the behaviour of complex structures comprising many elements where, for example, the exact sequence of element yield can significantly affect the overall seismic response. A need therefore remains for methods of dynamically testing or simulating whole-structure seismic response.

Shake table testing employs a rigid 'table' that supports the structure being tested and is dynamically excited by single or multiple actuators. In principle this can offer an accurate representation of real-world conditions, however it is hampered by major drawbacks. Testing at large scale requires very expensive, high-capacity actuation systems, resulting in the widespread use of scale models. However, the scaling of non-linear behaviour is fraught with uncertainty. In addition, accurate table control can be difficult to achieve due to interactions between the table and the specimen mounted on it, particularly when the latter responds nonlinearly. To negate the need for scaling and reduce the cost of dynamic test equipment, the PsD test method was developed; this allows slow, large-scale modelling of the stiffness elements of a structure, with dynamic terms computed numerically. If the structural response includes unpredictable rate dependent behaviour, for example due to a damper, then it may not be possible to compute all the dynamic terms reliably. In this case the test may need to be run at the correct loading rate in order to provide a realistic simulation; the RTHT and EFT methods were developed to meet this need.

The PsD or RTHT methods can be used to understand the performance of an entire structure. Alternatively, substructuring can be used to split the structure into parts whose behaviour can be modelled numerically with confidence, and those which really need to be tested physically. This approach enables a test engineer to understand the interaction between a critical structural element or component and the remainder of the structure. The genesis of different forms of hybrid testing can be seen in Figure 1. 


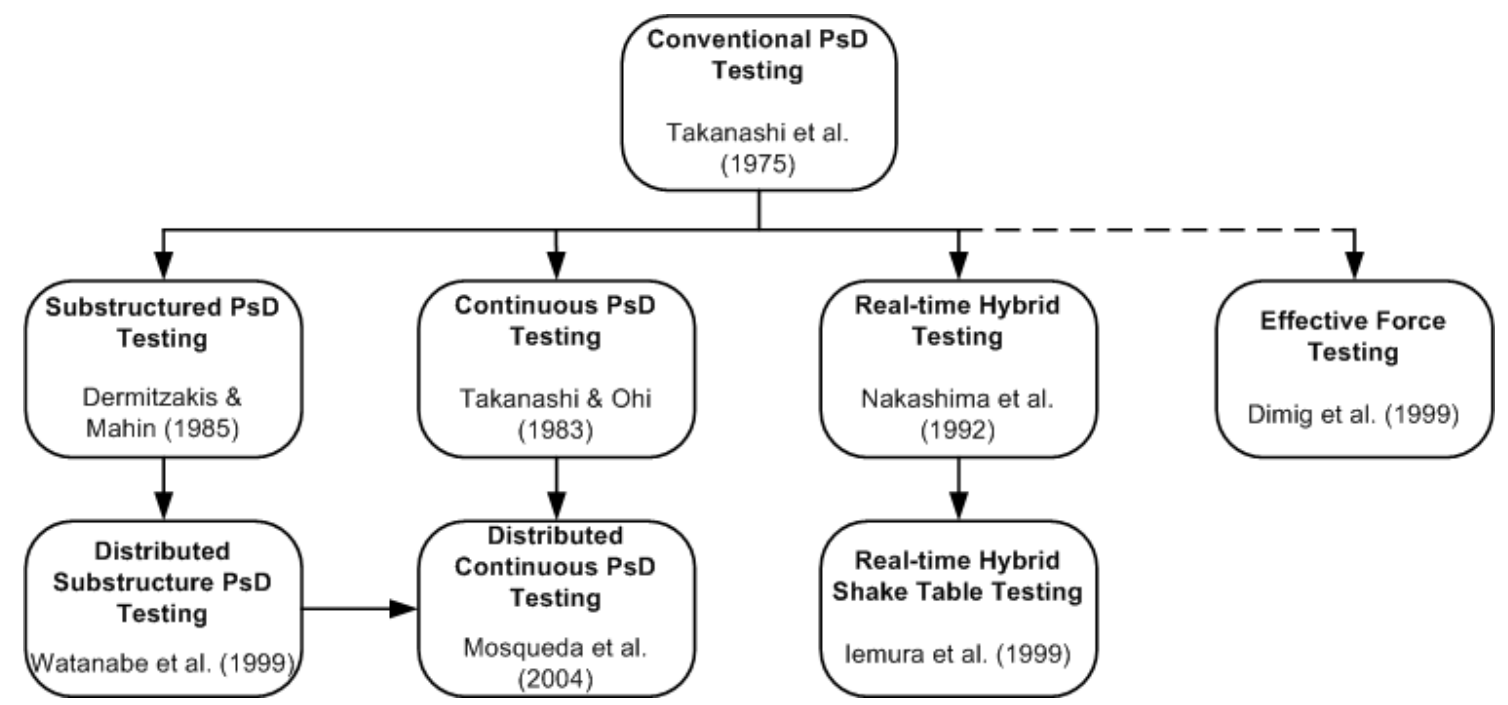

Figure 1. History tree of the types of hybrid testing and their first implementations (after [2])

\subsection{Conventional Pseudo Dynamic Test Method}

The concept of the PsD test was first proposed by Hakuno et al., [3] in which an actuator excitation of a single-degree-of-freedom (SDOF) system was combined with the solution of the equation of motion on an analogue computer. The PsD test method was first formally implemented by Takanashi et al., [4]. The method was seen a necessity to form more accurate analytical models to capture stiffness and strength deterioration during earthquake loading. The PsD method therefore analysed the nonlinear response of a structure during an earthquake, but with the stiffness nonlinearity modelled by a physical test specimen rather than an analytical model. The structural stiffness of the test member input into the computer model was obtained from the physical experiment of the structure. The predicted deformations computed by a piecewise linear method were then sent back to the actuator to give the next time step target displacement for the actuator to apply to the test system. This procedure was repeated until the end of the test.

A PsD test offers a powerful way of verifying the performance of seismic-resisting structural systems, and can provide valuable data for the development and calibration of nonlinear numerical models of structures and elements (see Section 6.1 for examples). It enables realistic seismic simulation without the need for dynamically rated test equipment, and the slow rate of testing allows for very complete monitoring and inspection of the state of the structure at every stage of the earthquake. 


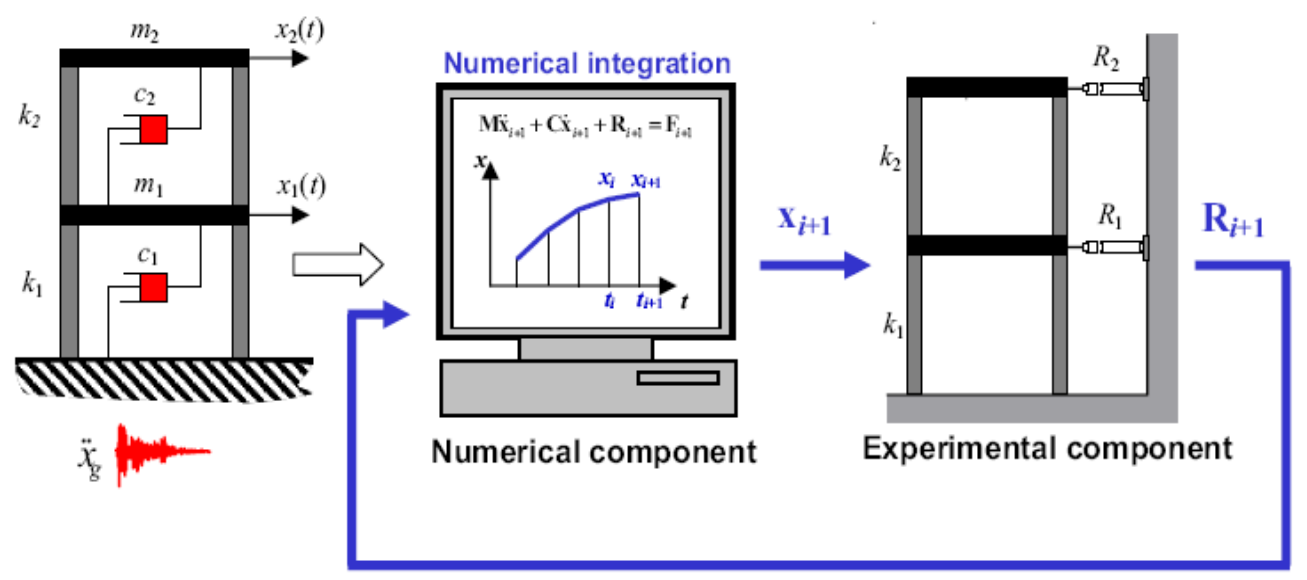

Figure 2. Schematic of the pseudo-dynamic test method [2]

In the PsD test method, it is conventionally assumed that the structure can be represented by lumped masses. At the location of the lumped masses, actuators apply the dynamic loading to the structure in the form of target displacements whilst inertia and damping are numerically modelled. The equation of motion in Equation 1 is solved over a series of time steps $\Delta t$, i.e. at the $(i+1)$ th step of:

$$
\mathbf{M} \ddot{\mathbf{x}}_{i+1}+\mathbf{C} \dot{\mathbf{x}}_{i+1}+\mathbf{R}_{i+1}=\mathbf{F}_{i+1}
$$

in which $\mathbf{M}$ is the mass matrix, $\ddot{\mathbf{x}}_{i+1}$ is the nodal acceleration vector, $\mathbf{C}$ is the damping matrix, $\dot{\mathbf{x}}_{i+1}$ is the nodal velocity vector, $\mathbf{R}_{\boldsymbol{i}+\mathbf{1}}$ is the restoring force vector and $\mathbf{F}_{\boldsymbol{i}+\mathbf{1}}$ is the external excitation force applied to the system. For a linear elastic structure $\mathbf{R}_{i+1}=\mathbf{K} \mathbf{x}_{i+1}$ in which $\mathbf{K}$ is the elastic stiffness matrix and $\mathbf{x}_{\boldsymbol{i}+\mathbf{1}}$ is the nodal displacement vector; for a non-linear system with hysteresis, the elements of $\mathbf{K}$ will change during the loading event and the relationship between $\mathbf{R}$ and $\mathbf{x}$ becomes more complex, depending on the displacement history as well as the current state. Figure 2 indicates how the displacement $\mathbf{x}_{i+1}$ is calculated by the numerical component and sent to the experimental test structure. The displacement command for the current time step is applied to the structure by the actuators. The measured restoring force, $\mathbf{R}_{i+1}$ is then fed back to the numerical model where the displacement for the next target displacement command is calculated. Effectively, the PsD test method is a computational dynamic analysis in which the stiffness term is measured physically [5].

In PsD testing, the slow loading rate of the test structure is required so as not to induce damping or inertia responses, because these have already been accounted for numerically [6]. In early investigations into the test method, issues of stability and accuracy of the test 
method were heavily researched ([7], [8] \& [9]). The assumed structural idealisation and numerical representation of the damping along with numerical integration errors raised concerns about the accuracy and stability of the test method (these are detailed in Sections 3 \& 4).

(a)

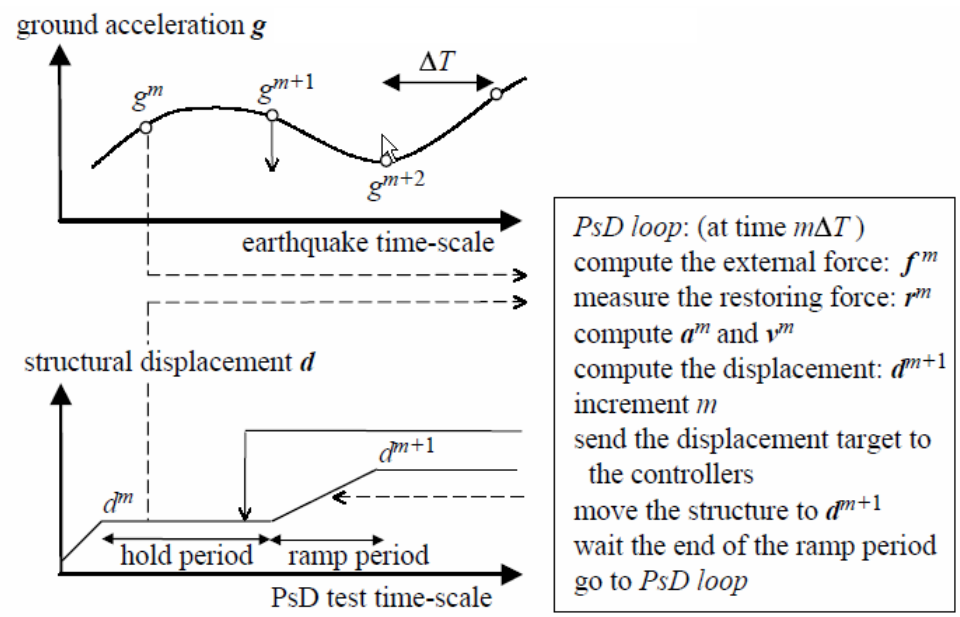

(b)

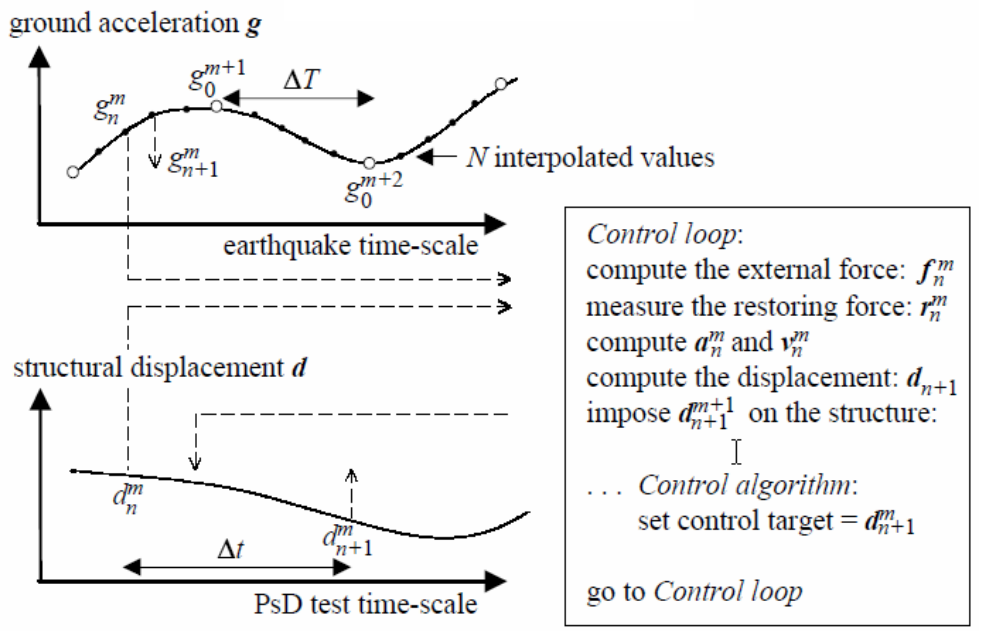

Figure 3. (a) Conventional pseudo dynamic method and; (b) continuous pseudo dynamic method (Magonette, [10])

\subsection{Continuous Pseudo Dynamic Test Method}

The PsD method is undertaken using an expanded timescale, allowing time required for numerical integration of the equations of motion and the implementation of the resulting command displacements by the actuators [11]. It is important that the response of the material being tested be rate independent as time dependent behaviour is not captured [10]. The time integration scheme employed needs to be efficient, therefore explicit integration procedures were typically used in early research. Some of the first implementations of PsD testing required a pause phase to allow for the computation of the numerical integration step to be completed, as can be seen in Figure 3(a). During the pause stage of the integration step, there 
is a certain amount of load relaxation that also leads to inaccuracies. The experiment should preferably be performed in a non-discrete manner to allow smooth loading in order to fully capture the response of the structure.

Early efforts at continuous hybrid testing met with limited success due to issues of overshoot in the algorithms used ([9] \& [12]). Figure 3(b) shows how the continuous PsD method developed by Magonette, [10] differs from the conventional PsD method. The control algorithm used by the servo-controller allowed the actuator to follow the target displacement accurately within small time steps preventing the need for the hold period. In addition to this approach, Nakashima et al., [13] and more recently Bonelli et al., [14], adopted an approach of parallel partitioned time integration in order to separately solve the equation of motion for the physically tested part of the structure and the numerical part of the structure (physical and numerical substructuring is described in Section 2.3). A fine time step is used on a small number of DOFs the physically tested part of the structure on one computer whilst a coarser time step is used on a larger number of DOFs [14]. The approach allows smooth continuous PsD testing.

\subsection{Substructured Pseudo Dynamic Test Method}

The important concept of substructuring in PsD testing was first introduced by Dermitzakis and Mahin, [15] and subsequently implemented by others; [16], [17], [18], [19] \& [20]. The concept of substructuring allows the critical part of the structure (typically with a highly nonlinear response) to be physically tested (physical substructure (PS)) and the rest of the structure to be simultaneously numerically modelled (numerical substructure (NS)). The force and displacement from the actuator(s) are fed back to the numerical model where they are used to solve the next time step command as can be seen in Equation 2. Substructuring prevents testing of large, heavy and costly structures. In practice, it is difficult to apply gravity loads physically in a substructured PsD test and very such few tests have been performed; this was, however, achieved by Obata and Goto [183]. If gravity loads are not physically applied to the substructure then the second-order P- $\Delta$ effects are taken into consideration numerically by geometric transformation. However, the buckling capacity of members in compression (e.g. columns) and the collapse load may be overestimated when gravity loads are not physically applied. In a substructured PsD test, the equation of motion can be written in discretised form as:

$$
\mathbf{M} \ddot{\mathbf{x}}_{i+1}+\mathbf{C} \dot{\mathbf{x}}_{i+1}+\mathbf{R}_{N_{i+1}}+\mathbf{R}_{E_{i+1}}=\mathbf{F}_{i+1}
$$


where $\mathbf{R}_{N_{i+1}}$ is the numerical restoring force vector for the numerical substructure DOFs, where, for a linear numerical substructure, $\mathbf{R}_{N_{i+1}}=\mathbf{K}_{N} \mathbf{x}_{N_{i+1}}\left(\mathbf{K}_{N}\right.$ is the numerical substructure stiffness matrix and $\mathbf{x}_{N_{i+1}}$ is the numerical substructure nodal displacement vector) and $\mathbf{R}_{E_{i+1}}$ is the restoring force vector for the physical substructure DOFs, which is likely to be a non-linear function of the experimental substructure nodal displacement vector $\mathbf{x}_{E_{i+1}}$. In the analysis, $\mathbf{R}_{N}$ is now calculated numerically and $\mathbf{R}_{E}$ is determined experimentally. At each step, Eq. (2) is solved for the displacements $\mathbf{x}_{N}$ and those elements of $\mathbf{x}_{N}$ that are at the interface between the physical and numerical substructures are applied to the test specimen by actuators. The resulting interface forces $\mathbf{R}_{E}$ are then fed back as part of the input to the next timestep.

When substructuring the system under test, great care needs to be taken to ensure that boundary conditions are realistically simulated. If boundary conditions applied to physical test specimens are not consistent with those used in the numerically modelled part, then misleading results may be obtained. In addition, it is important that the boundary conditions at the interface between the physical and numerical substructure are represented as realistically as possible by the actuators applying the interface forces.

Developments in multi-axis substructure testing have also been made using multiaxial actuators with uni-directional rotation or three-dimensional rotational hinges ([21], [22], [23], [24], [25], [26], [27] \& [28]). The multi-directional displacements of the test structure are taken into account by co-ordinate transformation methods based on trigonometry.

Possible sources of error in substructured tests are discussed in section 4.1.

\subsection{Real-time Hybrid Test Method}

Nakashima et al., [29] present the first system capable of performing a real-time (or fast) PsD test. In a RTHT, the numerical substructure accounts for the mass, damping and stiffness of the numerically modelled part, the damping and inertia response of the physical substructure are measured in the test along with the stiffness forces, and together these comprise the force feedback to the numerical model. For structures with components whose response has velocity dependent characteristics, the RTHT method is especially useful. In structural dynamics, the use of visco-elastic material or damping components, whose dynamic response is influenced by the applied velocity rather than just the inertia forces, is increasingly common. Section 6.2 presents examples of RTHTs on structures with velocity dependent 
components such as dampers. In a substructured RTHT test, the equation of motion can be written in discretised form as:

$$
\mathbf{M}_{N} \ddot{\mathbf{x}}_{N_{i+1}}+\mathbf{C}_{N^{\prime}} \dot{\mathbf{x}}_{N_{i+1}}+\mathbf{R}_{N_{i+1}}+\mathbf{R}_{E_{i+1}}^{\prime}=\mathbf{F}_{i+1}
$$

where $\mathbf{M}_{N}$ is the numerical substructure mass matrix, $\ddot{\mathbf{x}}_{N_{i-1}}$ is the numerical substructure

nodal acceleration vector, $\mathbf{C}_{N}$ is the numerical substructure damping matrix, $\dot{\mathbf{x}}_{N_{i-1}}$ is the numerical substructure nodal velocity vector and $\mathbf{R}_{N}$ is the vector of numerically computed stiffness forces. The vector of experimental fed-back forces $\mathbf{R}_{E_{i+1}}^{\prime}$ differs from $\mathbf{R}_{E_{i+1}}$ in Eq. (2) because it includes the inertia and damping forces from the test specimen in addition to the stiffness forces. In a typical substructured RTHT of a seismic structural damping device the experimental mass tends to consist of just the mass of the device under test, together with any moving elements of the test rig. The interface forces measured from the experimental substructure are fed back into the numerical substructure and used to calculate the target displacement at the next time step for the entire structure.

The challenge to overcome when a PsD test is run at a timescale close to or at realtime is that the command for the next time step from the numerical model needs to be computed very quickly. Nakashima et al., [29] completed a RTHT on a viscous damper. Nakashima and Masaoka, [30] developed a method to allow uninterrupted command signals in which previous command displacements are used to extrapolate the command displacement to its next value one time step ahead of the current time. Early examples of RTHTs include; [30], [31] [32], [33], [34] \& [35]. As noted by Bonnet, [5] these test arrangements were typically bespoke to the particular laboratory in which they were undertaken.

\subsubsection{Real-time Hybrid Shaking Table Testing}

Shake tables and actuators have been combined to perform RTHT (termed as a real-time hybrid shaking table test (RTHSTT)). Users of shake tables have seen this development as a positive one because, by physically testing only a part of a structure, a larger scale can be used; this can reduce the difficulties associated with using large scale factors in some shake table tests. RTHSTTs are most often undertaken on velocity dependent substructures or damping devices as the test records true dynamic behaviour. A commonly-cited target application is the use of tuned mass damper-type devices to reduce building response to extreme dynamic loads. These devices are generally most effective when positioned in the 
top storey of a structure. Using RTHSTT, this storey including the device can be tested at large scale, with the lower storey responses modelled numerically and the shake table providing the interface floor displacements.

The RTHSTT method is slightly different to a RTHT because the shake table dynamics have to be compensated for. Many researchers have undertaken RTHSTTs such as; [36], [37], [38], [39], [40] \& [41]. Iemura et al., [36] and Igarashi et al., [37] developed the first RTHSTT procedure to evaluate active and tuned mass damping devices, respectively. The damping devices formed the PS and the entire structure formed the NS. Igarashi et al., [37] identified delay in the shake table hardware as critical to ensure stability of the test system. The delay results in phase lag between the control signal and shake table response and is compensated for by digital filtering of the target displacement using a least squares approximation in the frequency domain.

Horiuchi et al., [38] present a delay response algorithm for a RTHSTT that uses the absolute acceleration response at the boundary between the NS and PS rather than the relative displacement response typically used in previous RTHTs. Rather than calculating displacements, a linear acceleration method of time integration is required to calculate the shake table commands. Reinhorn et al., [42] describe a small scale RTHSTT using force controlled substructuring with a flexible element (spring) being introduced between the actuator and test specimen to account for force noise. Shao et al., [41] describe the RTHSTT of a one-third scale three-storey structure tested using the same force-based shake table testing platform as Reinhorn et al., [42]. Lee et al., [43] evaluated the vibration control effect of a tuned liquid damper (TLD) for a building structure using the RHSTT method. An inverse transfer function of the shaking table measured from the relative table acceleration to the reference signal was used for control and to cancel out the dynamic characteristics of the shake table [43].

\subsection{Effective Force Test Method}

Effective force testing (EFT) is a conceptually different method for undertaking dynamic tests ([44], [45] \& [46]). The EFT method was developed in order to circumvent some of the issues associated with PsD testing. It had been previously noted by several authors ([6], [7] \& [47]) that a PsD test with explicit time-varying forces imposed at each lumped mass to conduct real-time tests could be implemented without the need for computing and imposing required displacements [44]. In the EFT method, the forces applied to the test structure are the products of the lumped masses and their accelerations, and are therefore independent of 
structural properties such as stiffness and damping. The EFT forces are known a priori for any given ground acceleration time history because the force is a calculation of mass multiplied by the acceleration. The benefit of the EFT method is that it removes the need for real-time computation of structural response. However, in order to achieve the correct dynamic response in a pure EFT test, the full structural mass needs to be modelled physically, which is often impractical. Therefore, without substructuring, its application is likely to be limited.

As with RTHT, control in EFT requires high quality controllers and servo-valves [47] hence, it was not until technical developments improved that Dimig et al., [44] could conduct the first EFT. The force control algorithm developed by Dimig et al., [44] was tested on an SDOF spring-mass-damper system. Errors were observed when the actuator was unable to excite the SDOF structure at its natural frequency due to the light damping of the structure and the interaction between the structure causing velocity feedback issues [44]. Shield et al., [45] performed an EFT on the same SDOF system as Dimig et al., [44], however, velocity feedback correction was applied.

Pan et al., [48] proposed a displacement-force mixed control method for EFT. A switching control method was used in which the actuator is controlled by force when the forces are large then controlled by displacement when the forces are small.

Zhao et al., [46] demonstrated the feasibility of the EFT method by comparing the nonlinear response of a single-storey steel framed structure with rigid diaphragm using both shake table and EFT methods. A nonlinear velocity feedback compensation scheme based on that by Shield et al., [45] with improved hardware was shown to be effective. However, they noted that, because actuators are putting energy into the system, there is a risk of instability if the compensation algorithm is not sufficiently accurate. The presence of structural damping within the test specimen offers some margin of error against instability and it may be that stability could be assured by artificially increasing the damping in either the physical or numerical part. However, such changes need to be made with care as they will reduce the fidelity with which the test represents real structural behaviour, arguably negating the purpose of the test.

Sivaselvan et al., [49] presented a new approach to hydraulic actuator force control using a displacement compensation feedback loop and added compliance of a flexible mechanism intentionally placed between the actuator and structure. The displacement compensation is needed to ensure control over the structure's range of resonant frequencies. The added compliance is required as the actuator is mechanically stiff but force control 
requires a mechanically compliant system to allow for small force adjustments. This problem is important in actuators that are designed as displacement control devices because they are particularly sensitive to force control parameters. Nakata and Kru, [50] are currently experimentally investigating the feasibility of a MDOF EFT.

Ultimately, any real-time testing system will be limited by actuator flow capacity, as increased model complexity is likely to result in higher structural velocities that need to be tracked by the actuators. This limit is particularly relevant to EFT, where the combination of large forces and high velocities requires very high oil flow rates which may approach the capacities of the servo-valves. The low mass present in most RTHT setups generally leads to lower force demands and hence achievable flow rates.

\section{ACCURACY AND STABILITY OF HYBRID TESTING - TIME INTEGRATION TECHNIQUES}

Very many numerical integration techniques for hybrid testing have been proposed, and it is not feasible to review them all here. A sample of the most commonly used algorithms is detailed here, with the aim of highlighting the main types of method available. These include the development of explicit, implicit and operator-splitting numerical integration schemes. See also [5], [51], or [52] for good overviews of numerical time integration algorithms in hybrid testing.

\subsection{Explicit Numerical Integration Techniques}

Explicit methods are often used in early implementations of hybrid testing because they are computationally efficient and easy to implement. The numerical integration scheme is termed explicit because the new response value for a step is calculated based on the previous step(s). One of the drawbacks to early implementations of explicit methods was their conditional stability, resulting in a limitation on the maximum time step that can be used. The maximum time step is limited by the natural frequency of the system. The limitation is defined as follows for the widely used central difference method (CDM); $\omega_{n} \Delta t_{\max }=2$, where $\omega_{n}$ is the highest natural frequency of the system and $\Delta t_{\max }$ is the maximum time step. The limitation is important for structures with higher modes of vibration [7]. However, in many applications, the NS can be represented by a simplified structural model that eliminates higher frequency modes. Therefore, simple and computationally quick explicit methods may be more appropriate. In the case of more detailed and complex NS, recently developed 
unconditionally stable explicit methods can overcome stability issues associated with higher frequency modes. The choice of integrator is clearly dependent on the application.

Many researchers have used the CDM as the integration scheme for PsD and RTHT ([9], [29], [30], [31], [32], [53], [54], [55], [56]). Wu et al., [56] observed that the CDM could be modified to provide explicit target velocity as well as explicit target displacement.

The Newmark method [57] and subsequent family of modified Newmark methods have proven popular in hybrid testing. Shing and Mahin, [7] have shown that error propagation properties of the Newmark method are better than those of the CDM. Thewalt and Mahin, [47] have also shown that the Newmark method is more reliable and superior to the CDM.

The Chang Method (CM) [58] is an unconditionally stable explicit algorithm that was extended by Chang [59] to be an unconditionally stable explicit method applicable to PsD testing without stability limits. The error propagation properties are better than the CDM and explicit Newmark method. The method has similar numerical properties as the implicit constant average acceleration method (CAAM) by derivation of coefficients based on the assumption that displacement and velocity are equivalent as those of the CAAM. However, the algorithm of Chang [58] was only unconditionally stable for stiffness softening and linear systems, whilst that of Chang [59] was only unconditionally stable for stiffness hardening systems. Chang et al., [60] overcame this by developing a new family of explicit algorithms for general PsD testing.

Chen and Ricles [61] developed an unconditionally stable explicit integration algorithm for RTHT using a discrete transfer function approach (known as the CR method). The method is explicit for both displacement and velocity. Chen and Ricles [61] detail the control theory used to select the algorithm parameters to achieve unconditional stability for a linear system. A linear ramp generator and extrapolation procedure ensures continuous servohydraulic motion. Chen and Ricles [62] extended this algorithm to include controllable numerical damping for RTHT.

\subsection{Implicit Numerical Integration Techniques}

Implicit numerical integration schemes calculate the new response value for a step based on one or more of the current response values as well as previous step values. Implicit algorithms were avoided in early research as they required iterative correction which could induce undesirable unloading and required the tangent stiffness of the system being tested, 
which can be difficult to evaluate during a test [63]. However, in contrast to explicit methods, implicit integration methods are in general unconditionally stable [11].

The Hilber-Hughes-Taylor (HHT) [64] numerical integration scheme ( $\alpha$-method) was developed in order to improve numerical dissipation to damp out spurious participation of higher modes. Numerical damping is beneficial for improving computational stability; however numerical damping also suppresses the accuracy of the solution. It is more important to minimise errors through the accuracy of the experimental instrumentation rather than relying on numerical damping to achieve solution accuracy through computational stability. The HHT scheme showed improved numerical dissipation of higher modes and is unconditionally stable when compared with the explicit Newmark method. Thewalt and Mahin, [47] were the first to successfully implement an implicit time integration scheme for PsD testing. The implicit algorithm was based on the $\alpha$-method developed by Hilber et al., [64] and was proven to be more reliable and superior to explicit schemes [65].

The value of two constants, $\gamma$ and $\beta$, in the Newmark method can be set such that the scheme becomes implicit and unconditionally stable. The method is most often applied as the CAAM with $\gamma=0.5$ and $\beta=0.25$. The CAAM does not produce any numerical damping, unlike the implicit Newmark method which provides numerical damping for $\gamma>0.5$.

A number of authors have developed implicit algorithms specifically for RTHT, namely; [52], [66], [67], [68] and [69].

\subsection{Operator-Splitting Techniques}

The implicit operator-splitting method (OSM) improves over other implicit schemes as it has a non-iterative approach. A procedure of splitting the numerical integration into implicitexplicit components was proposed by Hughes et al., [70] and first implemented in PsD testing by Nakashima et al., [16]. The method is implicit for the linear part of the response and explicit for the nonlinear part of the response i.e. uses the tangent stiffness of the test structure that negates the need for iteration.

In a similar fashion to the Newmark method, the OSM has had $\alpha$-damping applied to it, known as the $\alpha$-OSM ([16] \& [71]). The scheme was developed in order to combine the speed of computation from explicit methods with numerical dissipation. Nakashima \& Kato, [72] proposed an error compensation technique called the I-Modification that required the predictor stiffness of the structure and the difference between measured and command displacements [51]. Bursi and Shing, [65] showed that its use with the $\alpha$-OSM mitigates the effects of undershoot type experimental errors by introducing energy dissipation. 
Developments in the state space approach to time integration have been made by Wang et al., [73] and Zhang et al., [11]. Wang et al., [73] introduced a state-space procedure (SSP) for the OSM (termed OS-SSP). The state-space approach allows the equations of motion to be rewritten as first-order differential equations i.e. replaces a single second-order equation by two first-order equations. The method was shown to have enhanced stability and accuracy over the explicit Newmark method, although it was not unconditionally stable. Zhang et al., [11] developed a conditionally stable explicit modified predictor-corrector (MPC) time integration scheme using the SSP (termed MPC-SSP). The explicit nature of this algorithm allows it to be used on a linear system in a RTHT and has a similar performance as the OS-SSP method.

Bonelli and Bursi, [51] investigated the feasibility of implementing a predictorcorrector procedure into the generalised implicit Chung Hulbert method [74] (termed $\alpha-\mathrm{CH}$ ) for PsD testing. The method maintains the efficiency of the OSM but improves the accuracy by using the implicit predictor and secant-stiffness estimation of the actual structural stiffness. However, further developments are required for implementation in a RTHT.

When velocity dependent restoring force is considered in a RTHT, the OSM is effectively implicit. Therefore, Wu et al., [75] proposed an unconditionally stable predictorcorrector method based on the OSM that is explicit when applied in a RTHT.

Ghaboussi et al., [76] extended the explicit-implicit method of Hughes et al., [70] such that the damping matrix of the PS is not included as the energy is assumed to dissipate entirely due to material hysteresis damping. The authors provide guidelines as to the selection of parameters in the method of Hughes et al., [70] that relate to the quality of the predicted displacement and stability criterion. As the stability criteria are predefined, the method has the advantage of not requiring the initial stiffness of the PS.

Hung and El-Tawil, [77] developed a full operator method (FOM) that uses predicted accelerations to compute explicit displacement and velocity in the predictor step. The method is suitable in a quasi-static and RTHT. The advantages of the FOM over the OSM are the use of measured restoring force from the PS and the use of tangent stiffness as opposed to initial stiffness providing greater accuracy for highly nonlinear systems.

Hung et al., [78] presented an unconditionally stable implicit predictor-corrector algorithm termed the Predictor-Alpha-Corrector (PAC) method for quasi-static hybrid testing. The PAC method showed considerably reduced error compared to the OSM. 


\subsection{Integral Form}

In explicit/implicit time integration methods the fundamental second order differential equation of motion is solved, however an approach solving the integrated first order form of the equation of motion has been implemented by [79] \& [80]. Chang et al., [79] first proposed the scheme that is derived from Chen and Robinson [81], in which an incremental form of the explicit Newmark method is integrated. The equation of motion is now solved in terms of change in velocity rather than acceleration [80]. Using direct integration for PsD testing, cumulative errors may become large if the rapid variation of resistance of the test system is not captured accurately due to non-linear behaviour [82]. However, using the first order solution method errors resulting from the assumption that the structural properties remaining constant throughout a time step (linearisation error) are eliminated by the integration of the restoring force, while the sharp characteristics of the seismic loading are smoothed out by the integration of the applied force [82]. The result is an algorithm which is capable of picking up the effects of rapidly varying excitation forces and stiffness changes, while at the same time displaying improved error propagation characteristics over the standard Newmark explicit representation. The method showed improved ability to deal with rapidly varying loads and stiffnesses as often experienced in civil engineering structures. Algaard et al., [80] removed some of the drawbacks associated with the method namely; conditional stability and excessive numerical damping. As mentioned previously, Wang et al., [73] implemented the integral form using the state-space approach with the OSM resulting in a conditionally stable implicit algorithm. More recently, Kim et al., [83] presented a new convolution integral method (CIM) of time integration for a RTHT that addresses issues with size and complexity of the NS.

\section{ACCURACY AND STABILITY OF HYBRID TESTING - HARDWARE AND SOFTWARE}

Errors in PsD and RTHTs can be broadly separated into structural idealisation errors, numerical errors associated with the integration scheme (discussed in Section 3) and experimental errors. In particular, systematic experimental errors in hardware (actuator) have been the focus of significant investigation. 


\subsection{Errors in the Hybrid Test Method}

The structural idealisation used in a PsD or RTH test will have a significant impact on the accuracy of the method. The discrete numerical model used must provide a realistic representation of the mass, damping and (in substructured tests) stiffness of any numerically simulated parts. In a PsD test, there is a need to represent the mass of the structure by a small number of lumped masses, whose inertia forces can be replicated by a few actuators. Structures are not discrete entities, so the question arises; how well does the discrete model of the system represent the continuous system? Special consideration is therefore needed when performing a hybrid test to ensure the test specimen and test set-up can accurately replicate the proposed structure under consideration.

A particular area of importance is the interface between the numerical and physical parts of a substructured test. Again, it is usually desired to model this interface as comprising only one or two degrees of freedom, so that the necessary interface displacements and forces can be conveniently applied by very few actuators. The location and nature of this interface therefore needs careful thought, balancing realism against the pragmatism of what can reasonably be achieved in the laboratory.

As in any experimental or numerical modelling exercise, care needs to be taken over the boundary conditions. While fixed supports are easily applied within finite elements codes, providing complete fixity in the laboratory is difficult. If, for example, one was conducting a test involving notionally fixed-base columns in both the numerical and physical parts, failure to provide comparable levels of base restraint in each part could give misleading results. These problems can be minimised through preliminary static stiffness tests on the experimental substructure, and through updating of the numerical model based on feedback of the early experimental results.

Laboratory constraints dictate the complexity and size of the structure that can be tested. The question arises as to how much is the accuracy improved in performing a hybrid test? Kim et al., [83] developed an improved method of numerical integration that allows a complex and large NS to be analysed during a hybrid test. The issue of substructure selection was also addressed by Kwon and Kammula [84] and its influence on the accuracy of a hybrid test by developing a weighted systematic selection procedure for the NS. Yang et al., [85] addressed issues with complexity and size through an online optimising method for testing multiple identical substructures (e.g. bridge piers). In their tests, using an optimisation method, Yang et al., [85] updated material parameters of the numerically simulated bridge 
piers to match those of the experimentally tested bridge pier during a hybrid test. Spencer et al., [86] present a phased approach for hybrid testing to address issues of complexity. Accuracy and completion of the hybrid tests was ensured through a phased approach that proceeds through a series of increasingly complex analytical simulations followed by final large scale hybrid testing.

Abbiati et al., [87] observed that coupling between the PS/NS and the time integration scheme in a PsD test with a large number of DOFs can also cause instability issues. The PS time step is typically $2 \mathrm{~ms}$, but it is not possible in a PsD test to complete the NS analysis for a large number of DOFs within this time step. Abbiati et al., [87] addressed this issue of stability associated with large numbers of DOFs by using parallel processes for analysing the NS and PS separately. A paralleled partitioned algorithm was developed to perform time integration that allowed for subcycling to achieve continuous PsD testing.

Errors in experimental execution can be broadly classified as measurement or control errors. In particular, PsD and RTHTs are very sensitive to actuator control errors. Experimental errors are probably of most concern because the nature of the error can result in failure of the test procedure. The experimental errors can then be further sub-divided into either systematic or random errors [88]. The random errors are typically as a result of noise in the measurement system or round-off errors associated with the A/D conversion of the signal [88], but have been shown to be irregular in nature and produce negligible effects [89] when compared to systematic errors.

Systematic errors introduced by the actuator are of most concern in hybrid testing because they provide undesirable energy effects on the dynamic response of the system [63]. Due to the cumulative nature of numerical integration, incrementally small errors introduced to the system will result in resonance-like effects in the error growth. For higher modes, this resonance-like growth of cumulative error occurs faster than for lower modes. In MDOF systems, systematic errors will cause spurious higher mode response due to relatively small convergence errors. This issue was particularly problematic in early PsD tests, leading to a variety of mitigation strategies being developed. For example, it is possible to find the dominant frequency and tune the servo-control system to improve the actuator performance. Numerical integration with numerical damping can also help to reduce spurious higher mode effects.

In RTHT, a further significant issue is the unavoidable time delay between sending a command to an actuator and it achieving the commanded displacement. This has been the subject of extensive research, which is described in Sections 4.2 and 4.3 below. 
In PsD testing of 3D structures, measurement of multi-directional displacements is a non-trivial issue, since no established, reliable multi-direction transducer has yet been developed. For example, in Obata and Goto [27], a spatial truss-type multi-axial linear variable differential transformer (LVDT) arrangement was used, but this required an iterative transformation to determine orthogonal displacement components. The availability of a simple, accurate technique for 3D displacement measurement would make a significant enhancement to multi-axial PsD testing.

Errors associated with numerical integration have been discussed in Section 3; however determination of structural damping is not always straightforward. The exact characteristics of structural damping may be difficult to model because in reality different types of damping may exist e.g. viscous, Coloumb, etc [90]. Molina et al., [91] note that hysteretic damping depends on displacement history and is observed in the restoring force therefore viscous damping should not be included in the equation of motion in a conventional PsD test.

\subsection{Actuator Delay Compensation Techniques}

Hybrid testing is a complex closed-loop procedure that has a number of components within the system such as a computer, servo-controller, hydraulic actuator, test specimen and data acquisition system. The servo-hydraulic actuator can be thought of as a physical transfer system between the NS and PS. The servo-hydraulic actuator and its associated controller form a dynamic system, including a servo-valve (that controls the flow of oil to the actuator) and hydraulic piston (converts hydraulic energy into force/movement). Within the servohydraulic actuator, a time lag of the order of 5-10 ms exists between sending the command to the actuator controller and achieving the desired displacement of the test specimen. This time lag is often referred to as a delay. The delay can be reduced, but not eliminated, by careful tuning of the actuator's controller. In addition, it may not be constant throughout a test.

The impact of a time delay $\left(T_{d}\right)$ can be seen in Figure 4(a). The output displacement signal lags the input displacement command signal to the actuator (Figure 4(a)). The effect of this delay is the measured and actual restoring forces do not match. The reason for this is that the actuator has measured the restoring force before the actuator has reached the target displacement. The problem is that the algorithm being used to solve time integration assumes both the target displacement (command) and measured force (actual) correspond to each other. The hysteresis loop in Figure 4(b) shows that energy is added to the system at each command displacement timestep as a result of the difference in the measured and actual 
response. The delay has been shown to equate to negative damping in linear-elastic SDOF systems [32] and if this delay becomes larger than structural damping the response will diverge and become unstable [2]. This delay in the system is particularly important in fast or real-time hybrid testing as it can cause instability. Other less important delays also exist in the entire test system such as communication delays, computation delays and $A / D$ or $D / A$ conversion delays.

(a)

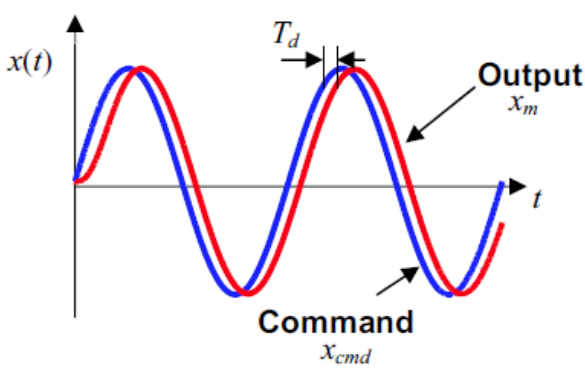

(b)

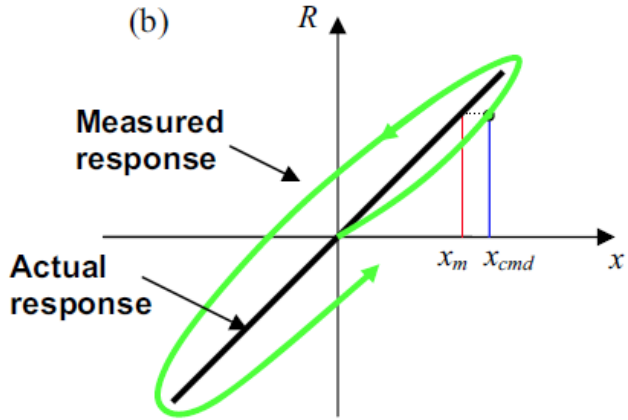

Figure 4. Effects of time delay on (a) displacement history; and (b) load versus displacement hysteresis [2]

The servo-hydraulic actuator, as part of the overall test system, needs to be controlled using, e.g., displacement feedback. The most common method for control of servo-hydraulic actuators is proportional integral derivative (PID) control. A PID controller is a feedback controller that uses a feedback loop to measure the error in a process and minimise it by adjusting the process control inputs. Proper control tuning of the actuator can help to mitigate errors associated with delay, however other methods of control of actuators (transfer systems) have been proposed that improve on PID control. Three main approaches have been developed to account for delay in RTHT systems; forward prediction schemes; performance evaluation schemes or model-based filtering. Bonnet et al., [92] provide a detailed description of compensation techniques up to 2005.

\subsubsection{Constant Delay Compensation}

The effect of the output and command displacements lagging each other was identified by Horiuchi and Konno [93] as a response delay. The time delay adds energy to the system because the force measured does not correspond to the command displacement. The effect of this time delay was first characterised by Horiuchi et al., [32] as being equivalent to negative damping for a linear-elastic SDOF system. Instability is caused by the negative damping being larger than structural damping. The method of delay compensation developed by 
Horiuchi and Konno [93] forward predicted the target response by a constant delay, $\delta t$, as shown in Figure 5.

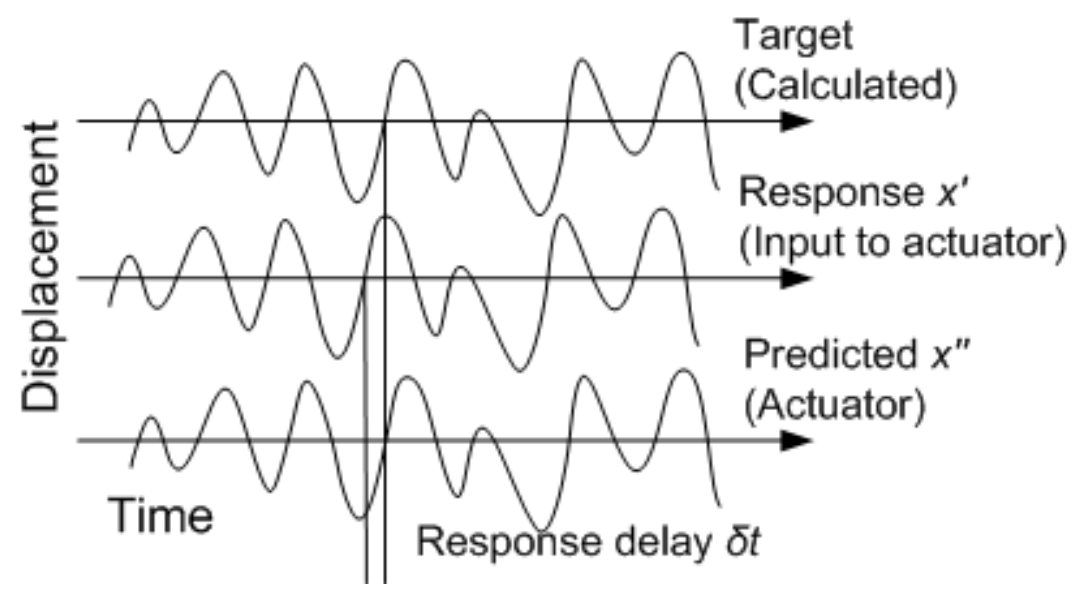

Figure 5. Schematic of the prediction method for actuator delay compensation [32]

Horiuchi et al., [32] developed a N-th order polynomial fit, where a third order polynomial was recommended in practice to forward predict the command displacement by one time step ahead of the current time step. The compensation method has been successfully implemented in a number of substructured RTHTs; [30], [31], [34], [94] \& [95]. Carrion and Spencer, [96] modified the forward extrapolation scheme of [32] to include the external excitation force and structural properties in a model-based approach.

Nakashima and Masaoka, [30] and Darby et al., [33] improved on the interpolation function's forward prediction scheme to more smoothly predict the displacement and velocity. Rather than the actuator moving at each time step, which is typically quite large, the interpolation schemes predict the output at sub-intervals resulting in a smoothed output. Horiuchi and Konno, [97] proposed a compensation scheme that predicts the acceleration based on the assumption that acceleration varies linearly. The predicted acceleration is then used to calculate the target displacement.

Zhao et al., [55] developed a phase lead compensator that removes the phase change caused by adjusting the compensation signal (in addition to the command signal) for a fixed time delay. Wallace et al., [98] developed a delay compensation technique that identifies the critical time delay for a linear system above which instability occurs. A delay differential equation is used to model the substructured system with a single fixed delay where analytical and numerical methods are used to determine stability. Delay differential equations are ordinary differential equations of the system with actuator delay explicitly included. Jung and Shing, [99] used PID control with a supplemental feed-forward scheme to compensate for delay, with the rate of change of the displacement command based on a fixed time delay. A 
Smith-type predictor [100] was proposed by Shao et al., [101] (also used by [49] \& [102]) to compensate for time delay in the displacement feedback. The Smith predictor achieves this by removing undesirable inner loop dynamics from the outer displacement feedback loop. Chen, [103] proposed a method called the inverse compensation method to compensate for delay in a system that modelled the actuator as a first order discrete transfer function. The model transfer function's inverse was then used to compensate for actuator delay [62].

Virtual coupling between the numerical and physical components was introduced by Christenson et al., [104] through virtual stiffness and damping to improve the stability of the system in the presence of time delay. The concept was first introduced by Colgate et al., [105] but also investigated by Seible et al., [106]. Virtual coupling improves performance by providing a trade-off between performance and stability depending on the relative restoring force in the PS.

\subsubsection{Adaptive Delay Compensation}

The assumption of constant actuator delay can lead to under- or over-compensation of delay resulting in experimental error when variable delays exist. Adaptive delay compensation is particularly required to ensure stability in a RTHT. Darby et al., [95] present a method of delay compensation based on adaptive control theory to deal with this issue. The delay estimation method uses a proportional feedback system to calculate the delay error for each time step; however the authors note that time to convergence is sometimes an issue and may not be efficient for rapidly changing nonlinear systems.

Wagg and Stoten, [107] developed outer-loop control using an adaptive minimal control synthesis (MCS) algorithm that worked in conjunction with the standard inner-loop PID control of the actuator. The method was demonstrated stable for continuous time, however Neild et al., [40] further developed the delay compensation scheme using an adaptive MCS controller for a RTHT. Control issues resulting from deleterious higher order numerical model dynamic effects were reduced by the use of filters on the displacement and acceleration signals. Stability was demonstrated on a shake table test of a one DOF massspring-damper system with the mass being the PS and the spring the NS. Lim et al., [108] demonstrated the application of a modified MCS outer-loop controller for a linear substructured two DOF system. The improvements made by Lim et al., [109] were to modify the demand of the MCS controller (termed MCSmd). The modification overcame the limitations of the MCS algorithm of Neild et al., [40] however, some complications imposed by the selection of initial gains still existed in the modified MCS algorithm. Bonnet et al., 
[110] used the MCSmd outer-loop control algorithm developed by Lim et al., [108] within a multi-tasking environment to perform a RTHT. The approach was demonstrated to perform well for substructured systems with stiff coupling. Tu et al., [111] developed an adaptive control algorithm based on the MCS algorithm for base isolation.

The adaptive polynomial based forward prediction algorithm proposed by Wallace et al., [98] used least squares polynomial extrapolation. The compensation method used an error driven adaptive feedback controller as an outer-loop control strategy. It was shown that the method allowed the forward prediction algorithm to accurately cope with frequency dependent system behaviour and operate with no prior knowledge of the system characteristics [98]. The approach built on the fundamental concepts of the Darby et al., [95] method of using the transfer system synchronisation error to ensure delay compensation [98].

Ahmadizadeh et al., [112] minimised variable actuator delay by incorporating a linear acceleration extrapolation scheme into the delay estimation algorithm developed by Darby et al., [31]. A learning gain achieved an online estimate of delay by comparing measured and desired displacements.

Chen and Ricles, [62] developed an error tracking adaptive compensation approach to deal with servo-hydraulic actuator delay in a RTHT based on the adaptive inverse compensation (AIC) scheme [103]. The AIC does not require an accurate estimate of the delay but an evolutionary variable is used to adjust the delay compensation based on a realtime evaluation of actuator control [113].

Chen and Tsai, [114] developed a combined adaptive second-order phase lead compensator (PLC) and an online restoring force compensator (RFC) for use in RTHTs. An adaptive gradient law is used in the PLC to estimate actuator delay during a test; however the adaptive gain needs to be tuned in the PLC. The RFC is computed using an averaged tangent stiffness that solves equilibrium of the equation of motion such that the structural response is corrected. Chen and Tsai, [114] investigated the effect on a SDOF system with low structural damping because the effect of actuator dynamics is more pronounced in such systems.

Wu et al., [115] present a method that nearly exactly compensates the time delay using upper bound delay and optimisation of feedback. The uncertainty of time delay is dealt with by an upper bound delay technique where the delay is assumed to be larger than the maximum possible delay i.e. is overcompensated. The displacement measurement is optimally selected so that the measured and actual displacements are as close as possible. The upper bound delay is calculated using the initial (maximum) stiffness, as the delay 
increases with increasing stiffness. The method reverts to an assumption of fixed delay if the real delay is greater than the assumed upper bound.

Chae et al., [116] developed an adaptive time series (ATS) compensator for RTHTs based on the relationship of input actuator displacement expressed in terms of the time series of the output displacement. The ATS compensator continuously updates the coefficients of the transfer system at each time step using least squares method to reduce the effects of time delay and amplitude error. Importantly, the adaptive gains do not need to be defined as per previous compensation schemes.

\subsubsection{Model-based Filtering}

In hybrid testing, very stiff structures introduce force errors even if the displacement is very accurate. A model-based filtering procedure to filter noise in a RTHT was introduced by Carrion and Spencer [2]. A model-based approach deals directly with the dynamics of the transfer system (actuator) rather than compensate for delay or time lag. A framework was developed by Carrion and Spencer [2] to filter restoring force and measured displacement noise from hybrid tests using model-based estimation and a Kalman filter [117]. The modelbased Kalman filter is employed because classical filters (e.g. low pass, bandpass or highpass filers) introduce an undesired phase lag on the filtered results, which is unacceptable in hybrid tests. System dynamics and inputs are taken into account in the filter estimation along with measurement and measurement error statistics [118]. The procedure was verified using a full-scale substructure PsD test in a two-bay single-storey steel frame [119] with significant improvement in measured force and moment versus analytical solution being observed.

\section{GEOGRAPHICALLY DISTRIBUTED HYBRID TESTING}

Hybrid testing by its nature is conducive to performing tests in geographically separate locations, as communication between the PS and NS can take place over the internet rather than through a fixed cable between laboratory equipment. Distributed tests are often referred to as on-line tests as communication between PS and NS takes place via the internet. A distributed test enables researchers to combine the capabilities of two or more sites [120]. This may add value by combining complementary facilities and expertise not available in a single laboratory - for example linking a foundation test in a geotechnical laboratory to a reaction-wall test in a structures laboratory. Splitting a test structure across multiple sites may also enable large-scale testing of bigger, more complex structures than can be tested in a 
single laboratory - for example, a substructured test requiring physical tests of multiple bridge piers may entail more space and equipment needs than can be met at a single test site. Of course, multi-site testing adds significant complexity and, in planning such a test, the researchers will wish to ensure that the additional benefits clearly justify the costs of the increased administrative and technical complexity.

The concept was first discussed by Campbell and Stojadinovic [121] and Watanabe et al., [122]. Watanabe et al., [123] introduced a test method using parallel PsD testing via the internet called Multi-Site Pseudo-Dynamic Substructure testing. However, the first successful distributed hybrid tests were conducted by Watanabe et al., [124] between Kyoto University (KU) and Osaka City University (OCU) (see also [125]).

Distributed hybrid tests have also since been conducted within the United States of America (USA) [119], UK [126] and Taiwan [127] and between a number of countries, notably Korea/Japan [128], the USA/Japan [129], USA/Taiwan [130], and New Zealand/USA [131]. The importance of collaboration and sharing of resources was identified formally by the George E. Brown Network for Earthquake Engineering Simulation (NEES) comprising of 15 advanced earthquake engineering test facilities. The NEES network combines testing facilities with computing resources to shift the emphasis of research from reliance on physical testing towards integrated experimentation, computation, theory, databases and model-based simulation [119]. A grid framework within NEES (NEESgrid) enables researchers to achieve this through telepresence, open access to consistent data via a data repository, access to open source analytical software and performance of distributed synchronised testing. NEES has inspired the inception of other similar research environments, for example; the Korea Construction Engineering Development Collaboratory Program (KOCED) [132], NZ-NEES [131] and UK-NEES ([126] or [133]). KOCED connected 12 large scale testing facilities at major universities around Korea. Uniquely, NZNEES provided a mobile command centre to collect in-the-filed data to be remotely sent back to the node. Within UK-NEES, a distributed hybrid test was performed between Bristol, Cambridge and Oxford Universities using a dedicated fibre-optic network.

\subsection{Development of Distributed Hybrid Testing}

The main frameworks that have been developed in distributed hybrid testing are; the Client/Server framework [124]; the Internet-based Simulations for Earthquake Engineering (ISEE) framework ([127] \& [134]) the Host/Station framework [135]; Networked Structural Laboratories (NetSLab) framework [136] University of Illinois - Simulation Co-ordinator 
(UI-SimCor) framework ([137]) and the Open-source Framework for Experimental Setup and Control (OpenFresco) [138].

\subsubsection{Client/Server Framework}

The Client/Server framework developed by Watanabe et al., [124] consists of a main computer and a number of local server/experimental systems (see Figure 6). The main computer controls both the dynamic analysis and the client system that controls the local server/experimental systems. The target displacements are transferred across the internet and controlled locally by each local server, with restoring force and measured displacements being sent back to the client. Two different configurations were adopted in the client/server approach, of which the more successful used Windows based workstations for all the main and local servers. The data communication over the internet was performed using the wellknown transmission control protocol/internet protocol (TCP/IP).

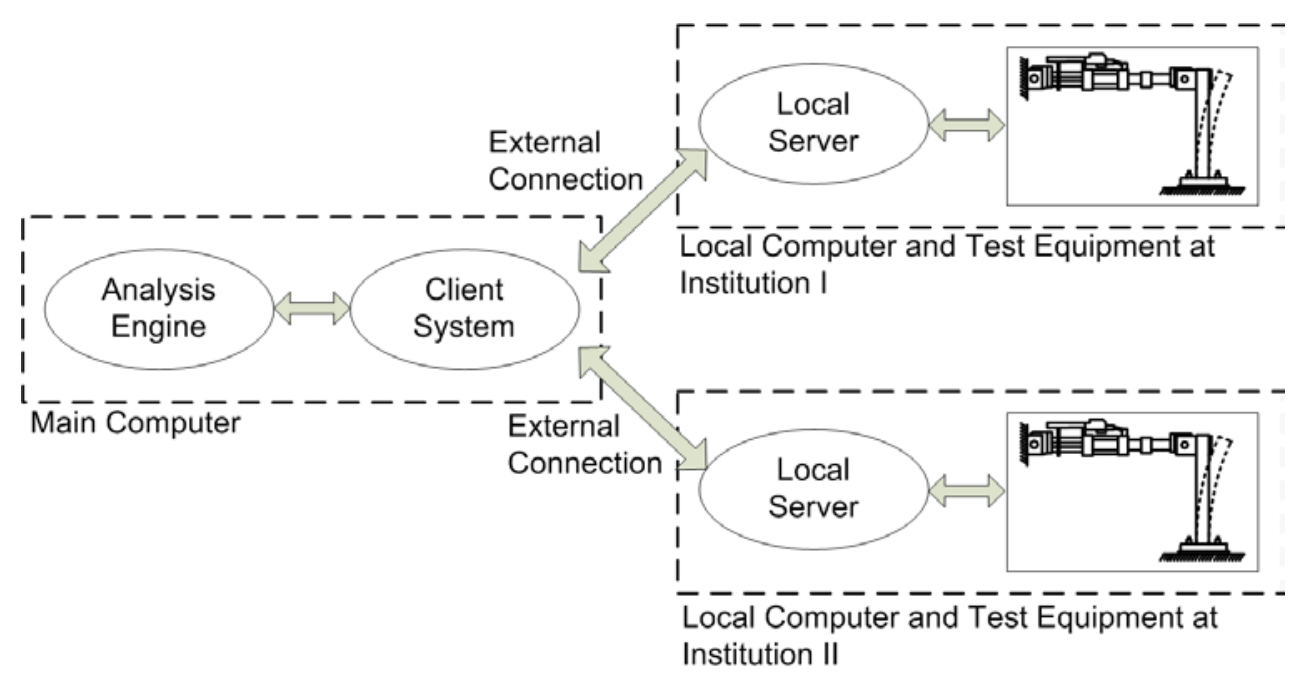

Figure 6. Schematic of client/server configuration and data communication (after [129])

Watanabe et al., [124] tested a steel and concrete piered viaduct with an average elapsed time per step of 22s between KU and OCU. A similar test of a base-isolated viaduct between KU and the Korea Advanced Institute of Science and Technology (KAIST) [128] took approximately $25 \mathrm{~s}$ for each timestep. The tests demonstrated the feasibility of the Client/Server framework, however the time dependent characteristics of the base isolator were not investigated.

Park et al., [129] performed distributed hybrid tests of a four span base-isolated bridge with the PSs located in KAIST and Korea Institute of Machinery and Materials (KIMM). Two data communication schemes were tested; one using a web based java monitoring system and the other using wireless internet phone technology. The mobility and encrypted 
data security of the mobile phone approach offer great potential for geographically distributed testing; however, data transmission speed was an issue when compared to a wired internet connection.

\subsubsection{ISEE Framework}

The ISEE is a client-server based framework that improved communication protocols compared to the Client/Server framework of Watanabe et al., [124]. Yang et al., [127] and Wang et al., [134] describe the development of ISEE through a database approach and application protocol approach, respectively (see also [130] and [139]). Yang et al., [127] developed the database querying approach to provide a platform for the exchange and warehousing of experimental data as shown in Figure 7. The structured query language (SQL) communication protocol controls the communication between the Analysis Engine, the Facility Controller and the Data Center. The use of SQL provides a ready-made platform for a web based data repository. The application protocol approach developed by Wang et al., [134] presents improved complexity in solving the data communication issues.

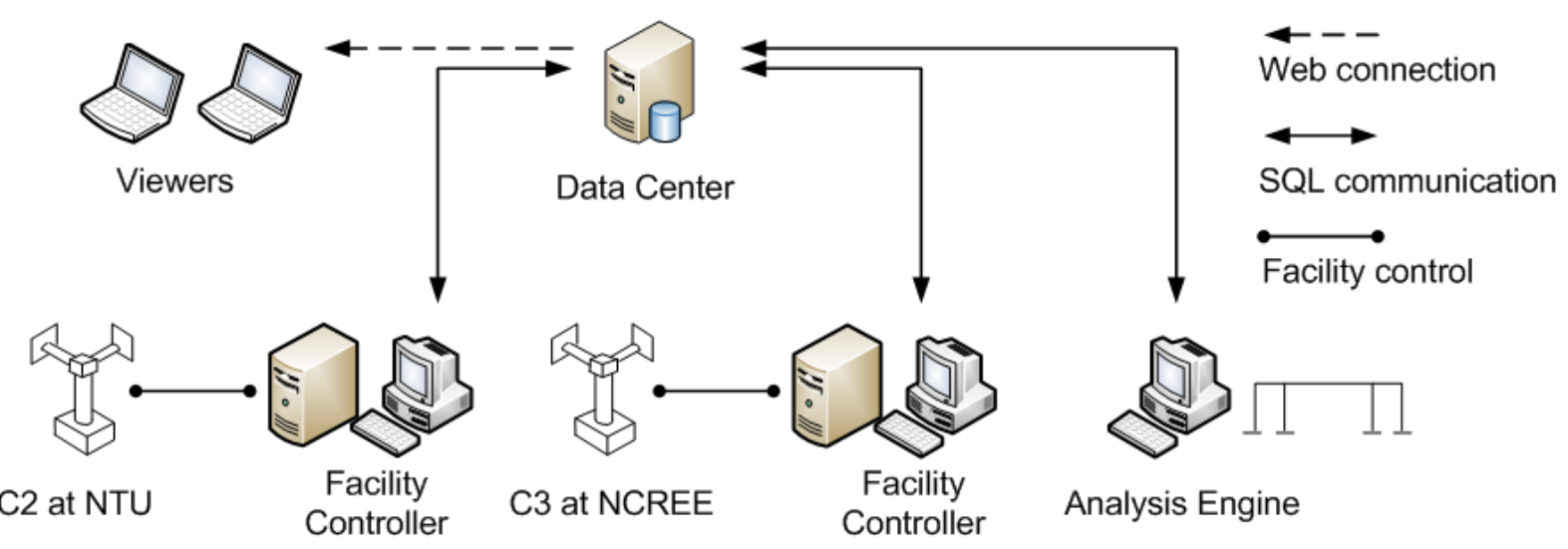

Figure 7. Network configuration of database approach for DSCFT test (after [139])

The ISEE database approach was applied to a double skinned concrete filled steel tube (DSCFT) hollow column from a single-storey, three-bay pinned structure tested between the National Taiwan University (NTU) laboratory and National Center for Research on Earthquake Engineering (NCREE) laboratory as shown in Figure 6. The application protocol approach was validated using a similar test and was shown to be slower but simpler to use in comparison to the database approach. Yang et al., [140] presented some further improvements on the ISEE system to include web broadcasting. 
(a)

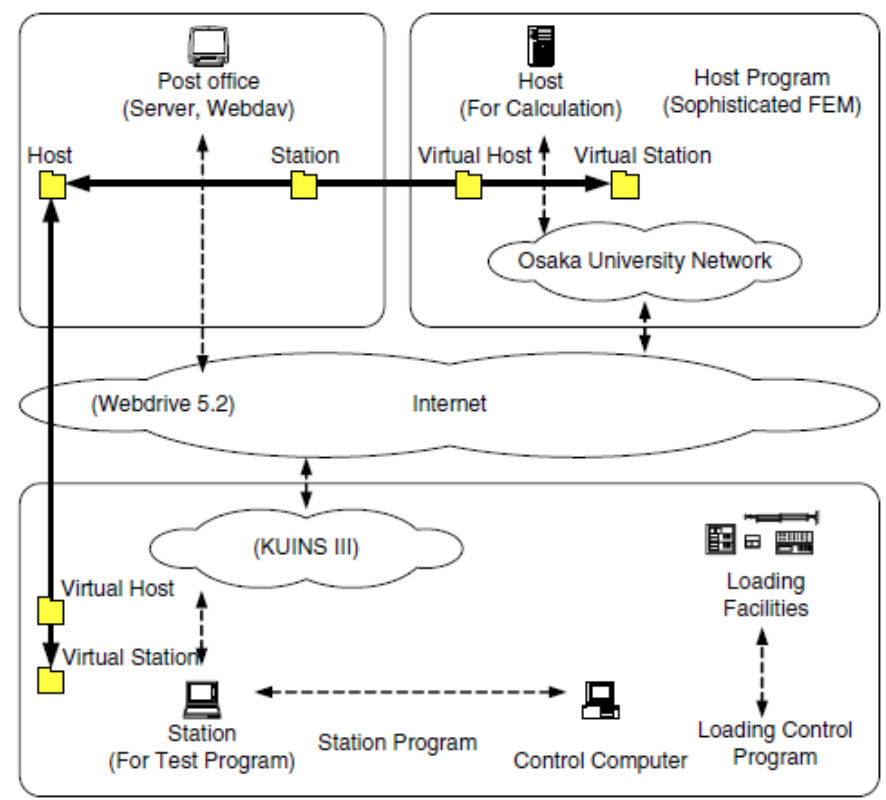

(b)

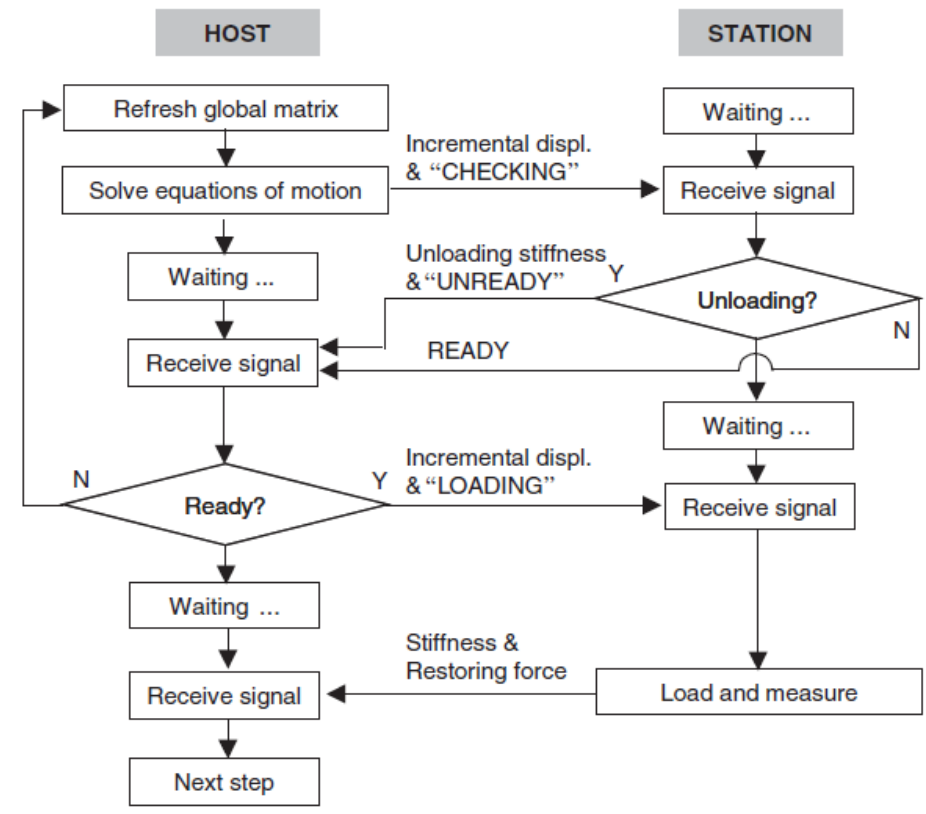

Figure 8. (a) Schematic of distributed hybrid test environment; and (b) flowchart of data exchange algorithm [135]

\subsubsection{Host/Station Framework}

The data exchange interface used by Tada and Kuwahara [141] was adopted by Pan et al., [135] in a client/server framework referred to as Host/Station here. Figure 8(a) presents a schematic of the Host/Station environment. The NS is simulated on the Host computer connected to the Osaka University Network, whilst the PS is tested in KU with the control run on the Station computer connected to the Kyoto University Network (KUINS III). The http protocol was used to connect the Station and Server computers and the data exchange was implemented using a dynamic link library. The data exchange procedure uses flags to 
apply the incremental displacements as shown in Figure 8(b), e.g. checking, loading, unloading, ready and unready.

An important contribution of this research was the implementation of distributed testing with a FEM program. Pan et al., [135] note that iterations for material nonlinearity in FEM packages are prohibitive in a distributed online test requiring a method to prevent such iterations. A method was developed to predict the tangent stiffness based on previous steps (using a least squares method), applying target displacement (using an implicit algorithm), measuring restoring force and correcting for difference between predicted and actual stiffness for the next time step. The method was shown to be successful at predicting the stiffness even for complex experimental hysteresis behaviour.

Pan et al., [142] improved on the approach of Pan et al., [135] by developing a distributed hybrid test method that treats the PS and NS as independent systems with the equations of motion for each substructure being calculated separately for each geographically separate location. Improvements in data exchange using a socket mechanism as compared to standard internet protocols were also developed. Wang et al., [143] \& [144] investigated some of the numerical characteristics of the test method developed by Pan et al., [142] in order to improve the stability and accuracy of the iteration scheme used by Pan et al., [142]. The method was capable of adopting FEM programs and dealing with nonlinearities, however performance was slow.

\subsubsection{UI-SimCor Framework}

[145] discussed a framework for distributed tests within NEESgrid called UI-SimCor. Pearlman et al., [146] and Spencer et al., [119] describe the Multi-site Online Simulation Test (MOST) of a two-bay single storey steel frame shown in Figure 9(a) that linked the PSs at the University of Illinois at Urbana-Champaign (UIUC) and University of Colorado, Boulder (UC) with the NS at the National Center for Supercomputing Applications (NCSA) in Urbana-Champaign. A control protocol (NEESgrid Teleoperations Control Protocol (NTCP)) provides remote access to the control systems of both the PS and NS. Transient problems such as network interruptions during a distributed test are accounted for by state transition of the control protocol using accepted, executing and terminated states. A set of requested actions (proposal) is sent by a client to the control protocol server. If the proposal is accepted, the action is executed by the client. If any site rejects a proposal, the entire test can be cancelled by the client. The NEESgrid data repository stores all experimental data (and metadata) and provides access to it, with data being archived incrementally during the 
running of an experiment. A simulation co-ordinator controls the PS and NS as shown in Figure 9(b). The MOST experiment lasted approximately 5 hours with 1500 time steps (12s per time step). The MOST experiment essentially used UI-SimCor to create an artificial separation between the simulated model and the time integration procedure [120]. Separately, Mosqueda et al., [147] made improvements to the NTCP for use within NEESgrid to reduce the the communication overhead for each integration time step.

(a)

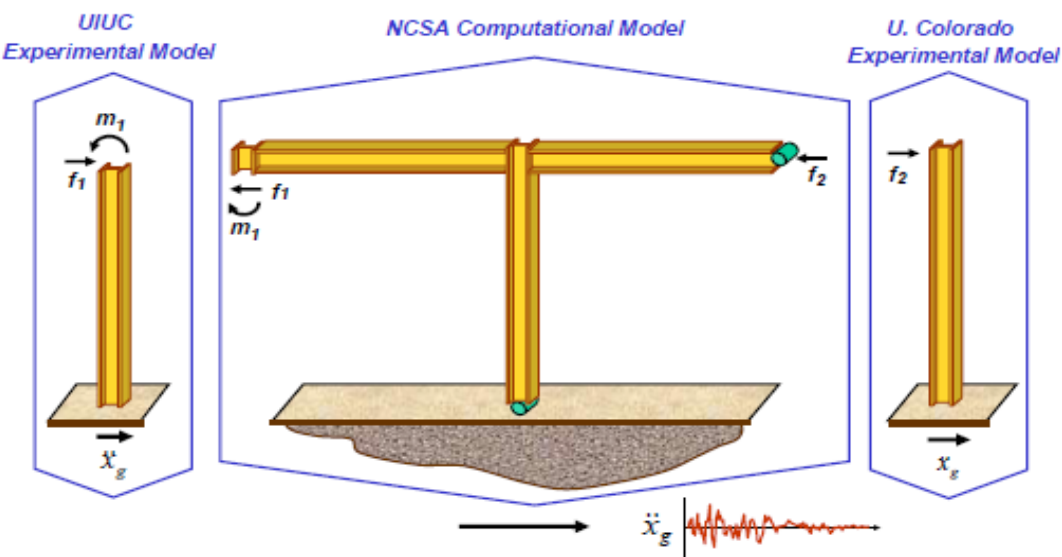

(b)

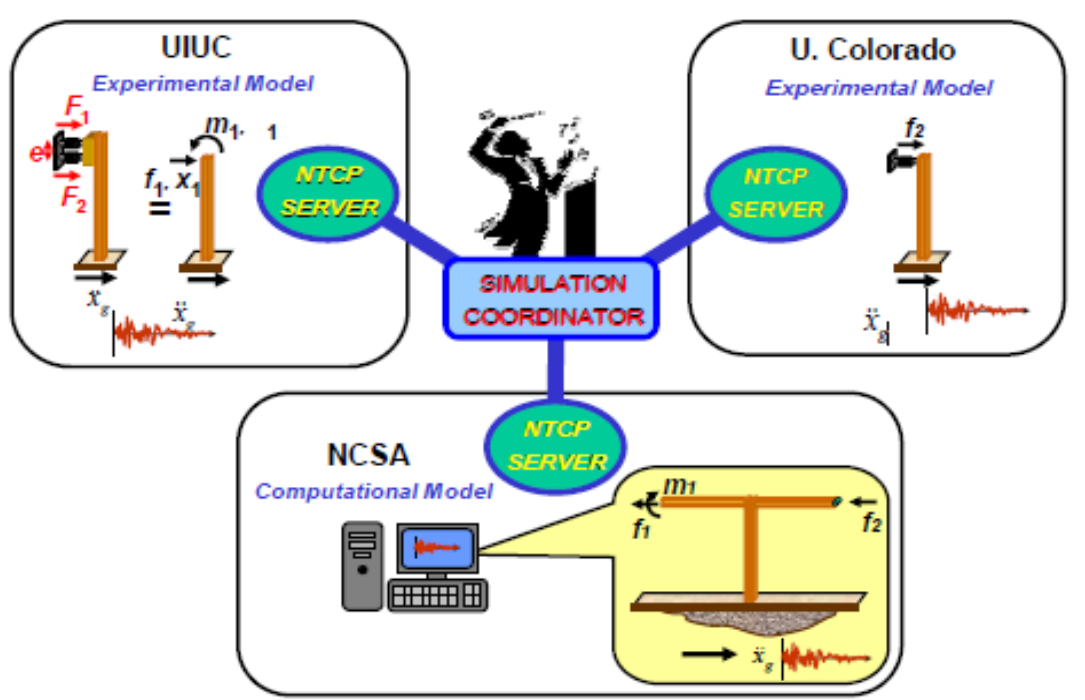

Figure 9. (a) MOST steel frame structure experimental set-up; and (b) modular framework for MOST experiment [119]

Kwon et al., [137] discuss the development of the software architecture that interfaces advanced analysis FEM packages, such as Abaqus [148], OpenSees [149], VecTor2 [150] or Zeus-NL [151] with UI-SimCor. Four application examples are discussed; a purely simulated soil structure interaction (SSI) bridge model, a purely simulated RC building model, a threesite distributed hybrid test termed Mini-MOST [152] and the MISST project [145]. 


\subsubsection{NetSLab Framework}

To facilitate research between the US and China the NetSLab internet-based network platform was developed. The network is based on the client/server approach with a socket communication mechanism as per Pan et al., [142]. NetSLab improves on the ubiquitous hierarchical internet infrastructure of firewalls and network address translators using the concepts of Dynamic Unified Data Packet (DUDP) and Generalized Data Communication Agency (GDCA) ([153] and [154]). The test results are processed by three types of tasks namely; Controller, Tester and Observer as shown in Figure 11. The Controller controls the test progress, data communication and performs the structural analysis. The Tester can be either Virtual or Actual. The Actual-Tester operates the test equipment in the laboratory and the Virtual-Tester can provide purely analytical results. The Viewer monitors and shares results. As shown in Figure 10, a centralised Controller PC with an Internet Protocol (IP) address co-ordinates the test participants (referred to as Testers) by sending and receiving requests using a Port 80 communication port.

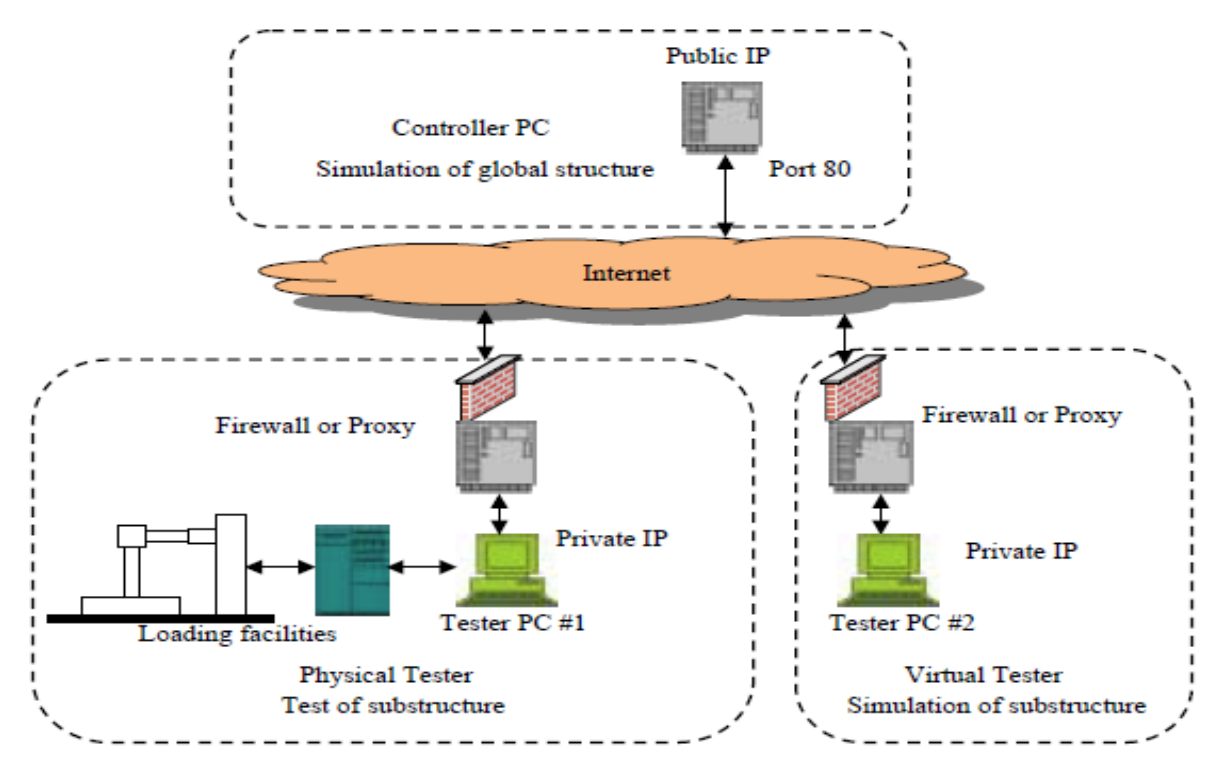

Figure 10. Schematic of communication strategy for remote hybrid testing in NetSLab [136]

The framework was developed for single storey structures (NetSLab-SDOF) only. Tests were carried out on a multi-span bridge $\left(1 / 5^{\text {th }}\right.$ scaled seven-column bent bridge [136] and $1 / 4$ scale four-span RC highway bridge [155]. In these tests, the PS was tested in either Hunan University or Harbin Institute of Technology (both in China) and the NS simulated elsewhere via the internet. NetSLab was shown to be feasible and reliable at performing distributed tests, however the method is limited as it only tests one dof and not multiple dof. The NetSLab capabilities were extended by $\mathrm{Xu}$ et al., [156] to allow remote structural health 
monitoring by sending measurement data from an on-site monitoring system rather than a test facility.

\subsubsection{OpenFresco Framework}

Within the Pacific Earthquake Engineering Research (PEER) Center there has been a drive towards the creation of a generic hybrid simulation framework. A client/server framework called OpenFresco [138] was developed with this goal in mind. The fundamental idea of OpenFresco is to allow testing to be undertaken at different laboratories, with different test equipment and without specialised knowledge required for the underlying software [157].

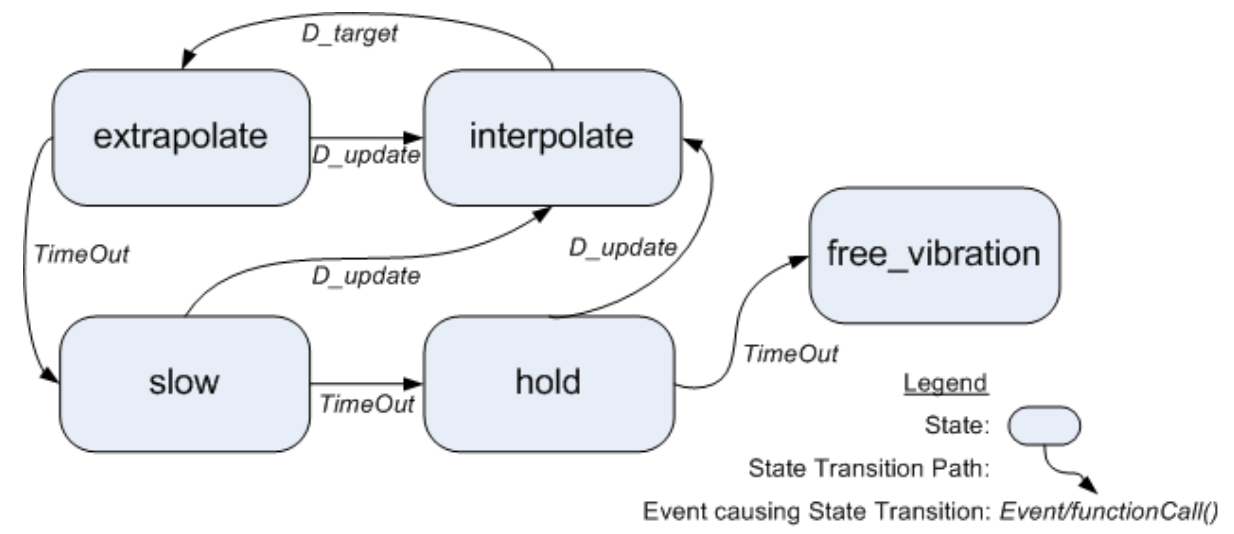

Figure 11. Event-driven strategy using polynomial predictor/corrector to generate continuous actuator commands (after [158])

A distributed test performed by Takanashai and Fenves [120] between UCB (client) and KU (server) evaluated the proposed object-oriented framework of OpenFresco. TCP/IP is used for network communication between the client and server. The power of this approach is that the classes defined in OpenSees can be used as the NS as OpenFresco utilises the objectorientated structure of OpenSees. The PS and its interface with the NS are dealt with by defining an Experimental Element class. This element provides the method to communicate with and control the PS and collaborates directly with OpenSees without a need for modification. A multi-tier software architecture approach is adopted in OpenFresco consisting of three tiers; a client tier (computational simulation), one or two middle server tiers (OpenFresco processes) and a backend server tier (laboratory control systems). Both local and distributed simulations can be performed using OpenFresco where ShadowExpSite and ActorExpSite are required on the client and server side respectively to undertake a geographically distributed test.

The middle tier provides the link between the computational modelling package and the data control/acquisition in the laboratory. Within the middle tier, classes are set-up to 
represent the real-world experimental element (ExperimentalElement), the real-world laboratory (LocalExpSite) and the ExperimentalSetup that transforms the trial displacements into actuator control displacements. Finally, the ExperimentalControl converts the trial displacements for the actuator into control signals for the respective control system in the laboratory. The benefits of OpenFresco are the pre-defined classes of experimental setups (e.g. OneActuator, TwoActuators,...), experimental elements (e.g. beamColumn, twoNodeLink, truss,...) and experimental control hardware (e.g. dSpace, xPCTarget, LabVIEW and SCRAMNet). The power of OpenFresco has been demonstrated by a number of authors; [120], [159] and [160].

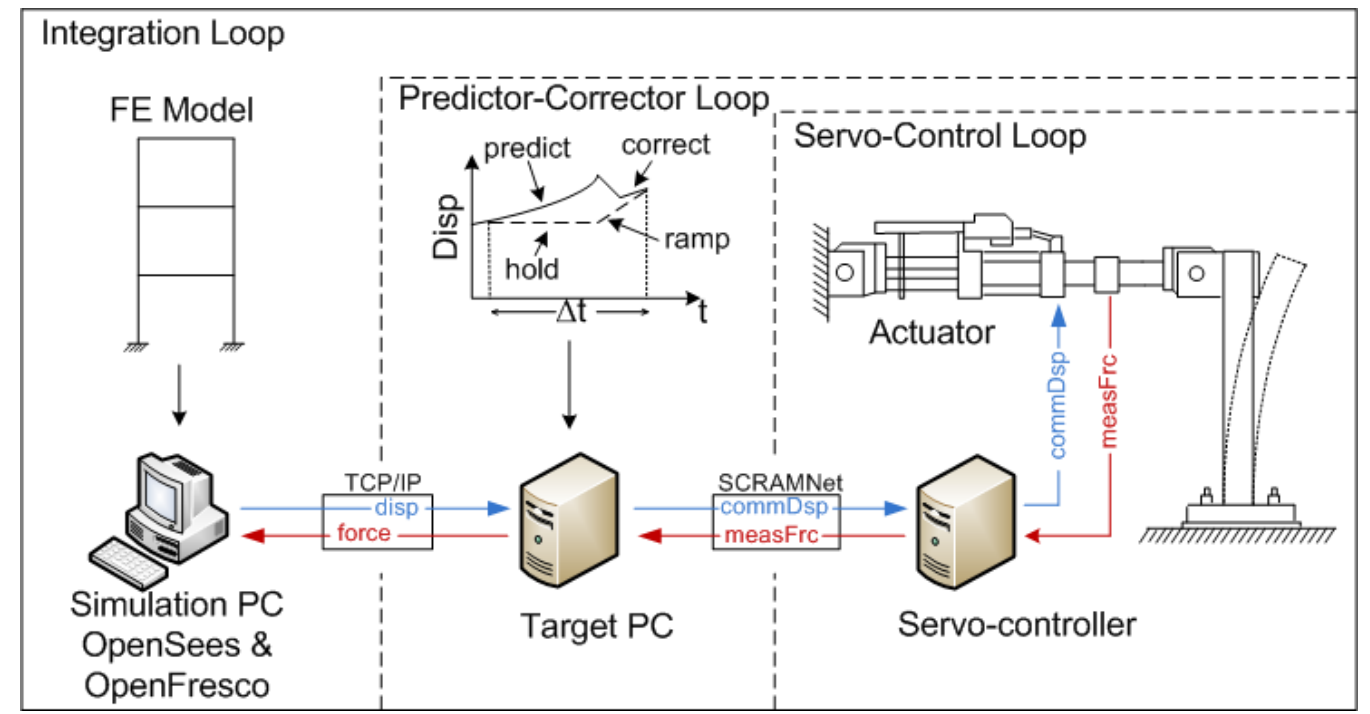

\section{Figure 12. Schematic of the multi-tasking control loops in the implementation of a hybrid test using SCRAMNet experimental control [161]}

The concept of an event-driven strategy to account for complexity and randomness during hybrid tests was developed by Mosqueda et al., [158] in which five states exist; extrapolate, interpolate, slow, hold and free vibration as shown in Figure 11. The default state is extrapolate, where the commands are predicted based on previously computed displacements and the integrator computes the next target displacement [89]. The state changes from extrapolate to interpolate after the next target displacement is received by the controller. The advantage of an event driven strategy is that logic can be used to handle excessive delays [162]. Excessive delays are overcome by slowing down the actuator to allow a command update. Alternatively, if the target displacement is not received after a specified number of steps, the state transitions to a hold state to allow the target displacement to be received. After this if no target displacement is received the system times out to free vibration.

Nakashima and Masoaka, [30] were the first to separate the integration of the equation of motion and signal generation into two separate tasks on a single processor. Schellenberg et 
al., [161] utilised this concept of multitasking to undertake tasks on separate processors (see [89]) to improve computational efficiency in distributed hybrid tests. Figure 12 indicates the three-looped control architecture used in OpenFresco to undertake hybrid tests. The outer control task is run on a separate local processor than the servo control loop. The intermediate control loop is termed the predictor-corrector control loop and allows synchronisation between the outer and inner control loops, which perform their tasks at different time scales.

\subsection{Recent Developments in Distributed Hybrid Testing}

Kim et al., [163] presented the first distributed RTHT of a scaled two-storey shear frame structure (NS) with MR damper (PS) tested between University of Connecticut and UIUC. Every 2ms data was sampled and the equation of motion of the NS was solved using explicit Runge-Kutta method because of its speed and lack of iteration. A Smith predictor-based approach accounts for network communication time delay within the simulation co-ordinator (UI-SimCor). Distributed RTHT error of approximately 5\% for peak displacement, peak damper force and energy dissipation of the MR damper was observed when compared to local RTHT results.

Hacker et al., [164] discussed improved distribution of test data through a repository called NEES Project Warehouse that manages the scientific data using web-based data analysis and simulation. The NEES Project Warehouse extends the framework of the centrally maintained web-based gateway called NEEShub [165]. The aim of NEEShub is to provide an accessible framework within NEES of uniform processes and data formats to enable greater collaboration and sharing in earthquake engineering.

More recently, Ojaghai et al., [166] demonstrated the feasibility of performing distributed hybrid tests in real time over the internet, through a series of experiments conducted between Oxford and Bristol universities in the UK. The tests used existing hardware and control systems at both sites, with modifications designed to minimise local delays and to prioritise real-time communications over other processes. Real-time hybrid testing was achieved across a variety of relatively simple test set-ups. However, the method was tailored to the particular laboratories involved and was not readily transferable to other sites. To address these limitations, Lamata et al., [167] \& Lamata et al., [168] have sought to develop a more structured framework, known as Celestina, to promote multi-site collaboration in earthquake engineering. This includes both the capacity for data sharing through a virtual database in which the local databases of participating institutions can be accessed as though they are part of a single, central site (Celestina-Data) and a robust 
protocol for the setting up and performance of distributed hybrid simulations (Celestina-Sim). The efficacy of the Celestina-Sim approach has been demonstrated through distributed simulations between Oxford (UK) and Kassell (Germany).

\section{APPLICATIONS OF HYBRID TESTING}

Much of the early research into PsD testing dealt with the accuracy, stability and reliability of the test procedure rather than the actual application of the test method. Only a small variety of the notable PsD and RTHT experiments that solely used the test method as a form of dynamic testing (rather than for development of the method) are discussed herein. Often, PsD and RTHT have been performed on SDOF systems. However, numerous examples of the application of PsD to MDOF structures exist, and several of these are discussed in Section 6.1. For RTHT, tests on MDOF physical substructures are rare, because the stiff coupling between multiple actuators linked via their connection to a single test specimen makes realtime actuator control extremely challenging.

\subsection{Applications of Pseudo Dynamic Testing}

Balendra et al., [169] performed one of the first PsD tests that detailed the application of the test as a research tool rather than focusing on the implementation of the test method. performed a PsD test on a full-scale, single-storey, eccentrically braced frame (without substructuring) with a shear link designed to dissipate energy. Test results were used to validate an analytical model. The PsD method was shown to adequately capture the inelastic deformation characteristics of the shear link.

Shing et al., [170] performed a substructured PsD test of a half-scale concentrically braced steel frame at UC. The bottom-storey braced frame formed the PS whilst the remainder of the three-storey braced frame structure formed the NS. Results indicated the importance of modelling the flexibility of the gusset connections accurately as the deformation of the connection has a significant influence on the seismic capacity of the frame.

Buopane and White, [171] performed a PsD test of a half-scale two-storey masonry infilled reinforced concrete framed structure. Accuracy of the dynamic actuators was provided by an iterative actuator control scheme developed by Seible et al., [106]. Intentional soft coupling as developed by Seible et al., [106] was used to overcome the spurious higher 
modes associated with PsD testing of stiff structures. Results demonstrate good seismic performance by maintaining compressive strut action in the masonry wall.

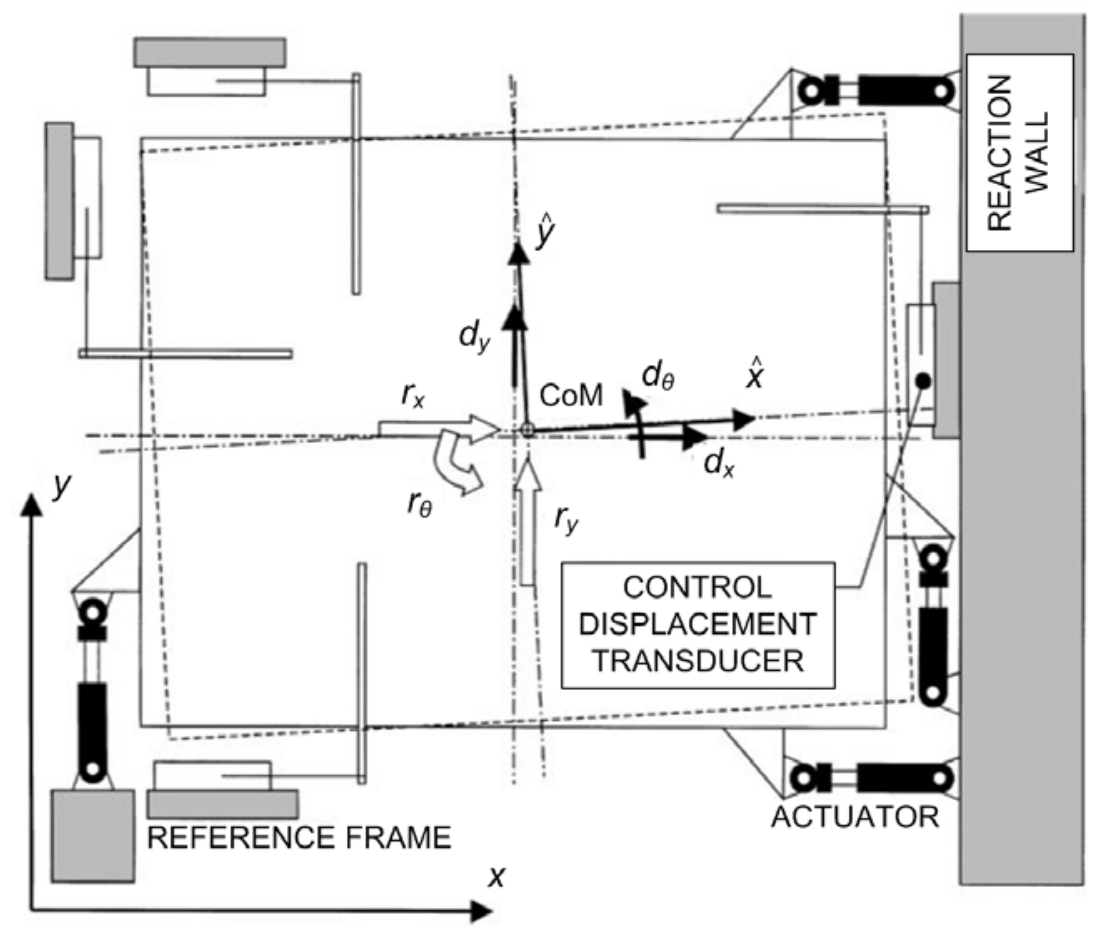

Figure 13. Generalised floor plan of bi-directional PsD test [22]

Molina et al., [22] performed bi-directional PsD testing of full-scale three-storey building at the Joint Research Centre, Ispra, Italy, as shown in Figure 13. The building was constructed of steel columns and beams with composite reinforced concrete floor slabs. The feedback displacement of each floor for control purposes was achieved through linear displacement transducers attached to each floor with geometric transformation of the target displacement being required. The results were used to investigate the influence of the slab on the seismic moment capacity of the beam-column connections.

Pegon and Pinto, [19] discuss general developments in PsD substructured testing of a full-scale bridge structure at the ELSA Laboratory at Ispra, Italy. Notably, the paper investigates the topic of asynchronous testing. The main complexity in asynchronous testing is that typically the entire structure being tested is fixed to a reaction floor and for an asynchronous test only physically unconnected parts of a test structure can be investigated. A unique and absolute reference frame is therefore needed to describe relative and absolute motion; however this requires great care deriving the expression of the coupling term appearing in the connecting dofs [19]. PsD tests of a RC bridge comparing synchronous and asynchronous input motion showed similar results for the shorter pier tested but substantially higher ductility demands in the medium length pier. 
Molina et al., [20] performed substructured PsD tests of a full-scale rubber base isolated four-storey building. The isolators formed the PS whilst the superstructure formed the NS. The tests were not performed at real-time. Therefore alteration of the restoring force was required to compensate for material strain-rate effects of the rubber isolators. Tests investigated the effect on response of two types of rubber isolators (soft-blend and mediumblend rubber).

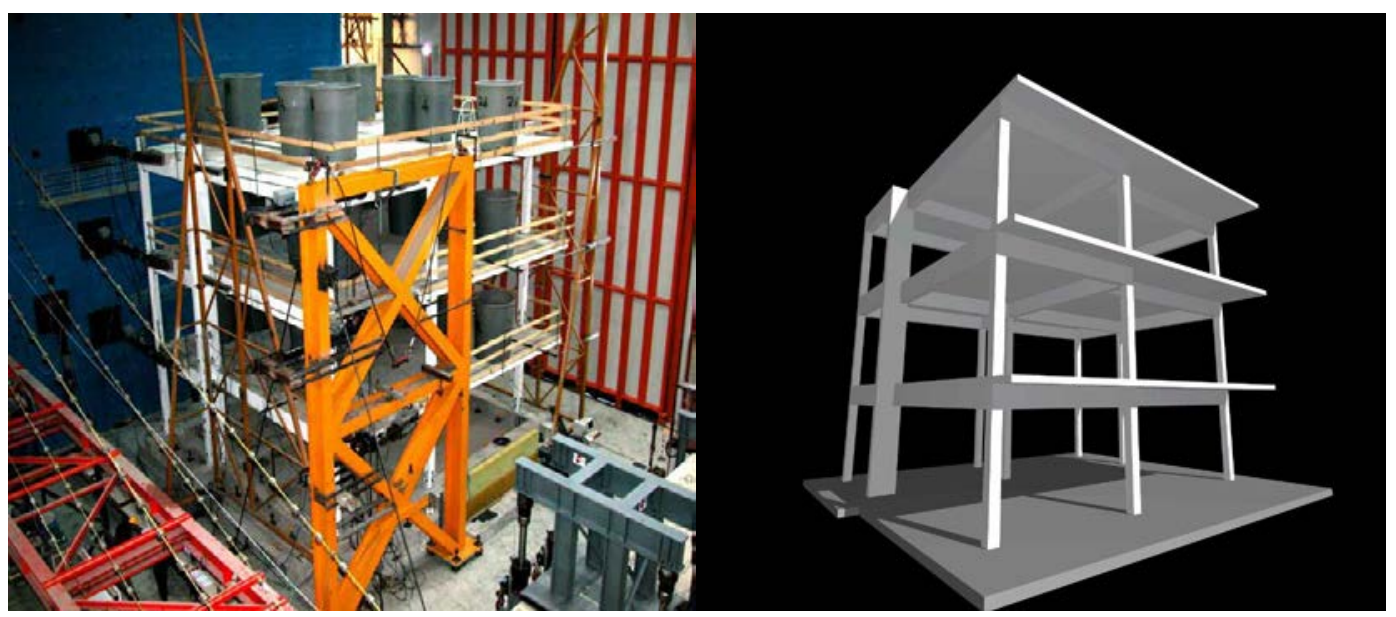

Figure 14. SPEAR structure (a) test setup and; (b) 3D view [172]

Pinto et al., [173] performed substructured PsD tests of a six pier model of an existing bridge. Two 1:2.5 scaled piers formed the PS and the abutments, four remaining piers and bridge deck formed the NS. Importantly, the weight of the deck was applied as vertical loading. Asynchronous input excitations were applied as per the method developed by Pegon and Pinto [19]. Three intensity ground motions were applied to test the vulnerability of the bridge and demonstrated the poor performance of the bridge. This test provides a particularly good illustration of the benefits of the substructuring approach, since it is unlikely that any laboratory in the world (either shake-table or PsD) could accommodate a test of the full structure at an acceptable scale.

Negro et al., [172] performed a full-scale PsD test on a torsionally unbalanced reinforced concrete framed structure, as shown in Figure 14. The structure tested in the Seismic PErformance Assessment and Rehabilitation of existing buildings (SPEAR) project has been extensively investigated (see also; [174], [175] and [176]). The building represents old construction types in southern European countries that were not subject to specific designed to be earthquake resistant. McCrum and Broderick, [177] also performed a series of full-scale substructured PsD tests on a torsionally irregular multi-storey steel concentrically braced frame structure. Both projects identified the significance of plan irregularity on seismic response. 
Paquette and Bruneau, [178] performed PsD testing of a full-scale one-storey unreinforced brick masonry specimen with flexible wooden diaphragm floor. The research goal was to better understand the flexible floor/rigid wall interaction of this typical type of construction. Even with excessive cracking observed, the building was found to be quite resilient to the applied earthquake loading.

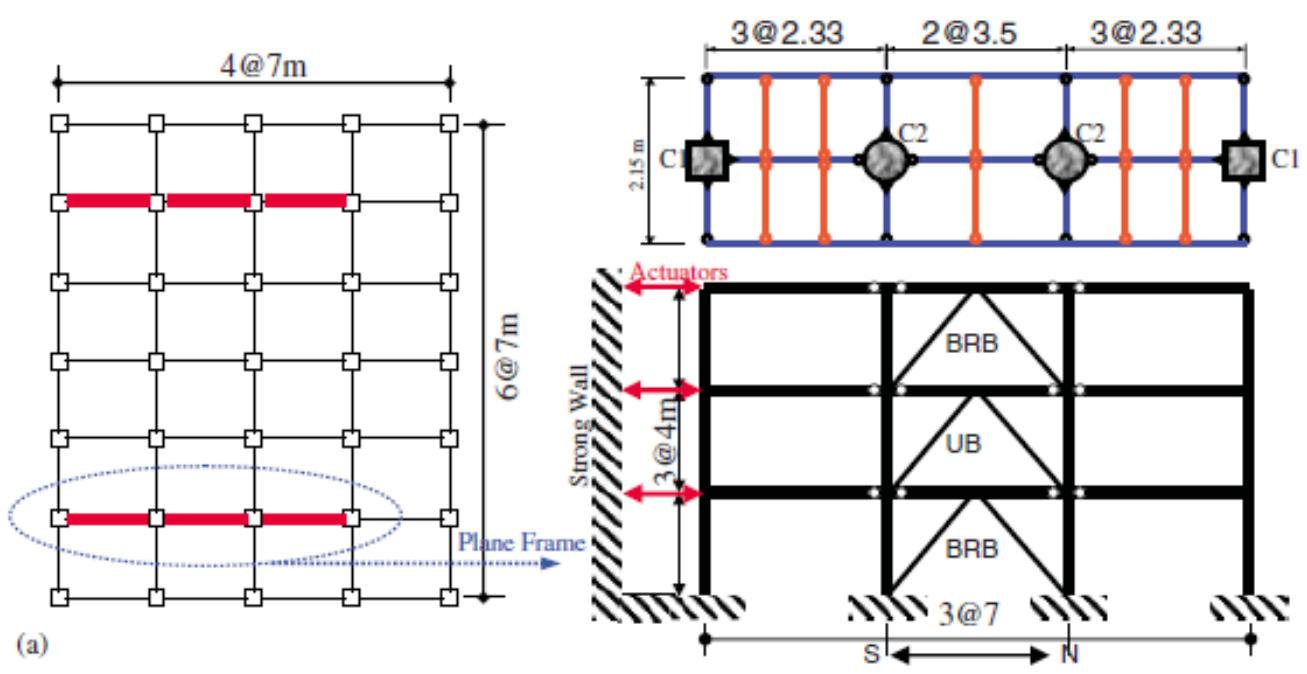

Figure 15. Dimensions and test set-up of the full-scale CFT/BRB composite frame [179] Tsai et al., [179] and Tsai and Hsiao, [180] describe a substructured PsD test of a full-scale three-storey three-bay concrete filled tube CFT/BRBF structure as shown in Figure 15. Second order P-delta effects in the columns were taken into consideration during the computation of the target displacement by modifying the restoring force. Many parts of the displacement based designed structure such as the gusset plate connections and seismic performance of the BRBs were under investigation during the test.

Eatherton, [181] performed half-scale substructured PsD tests of a controlled rocking steel braced frame system that eliminates post-earthquake permanent residual drifts. The tests took place at UIUC within the NEES network. A series of quasi-static hybrid tests were performed on scaled three-storey steel braced frames. Experimental results showed that the controlled rocking system satisfies the stated performance goals, with a predictable hysteresis and the displacement of the frames almost entirely due to rigid body motion.

Kammula et al., [182] investigated the performance of a self-centering energy dissipative bracing system using substructured PsD testing. Over thirty full-scale hybrid tests of a six-storey steel structure were carried out to derive seismic fragility curves. The first storey braced frame formed the PS whilst the remainder of the upper stories formed the NS. 
Test results demonstrated the reliability of the test method for seismic fragility assessment of structures.

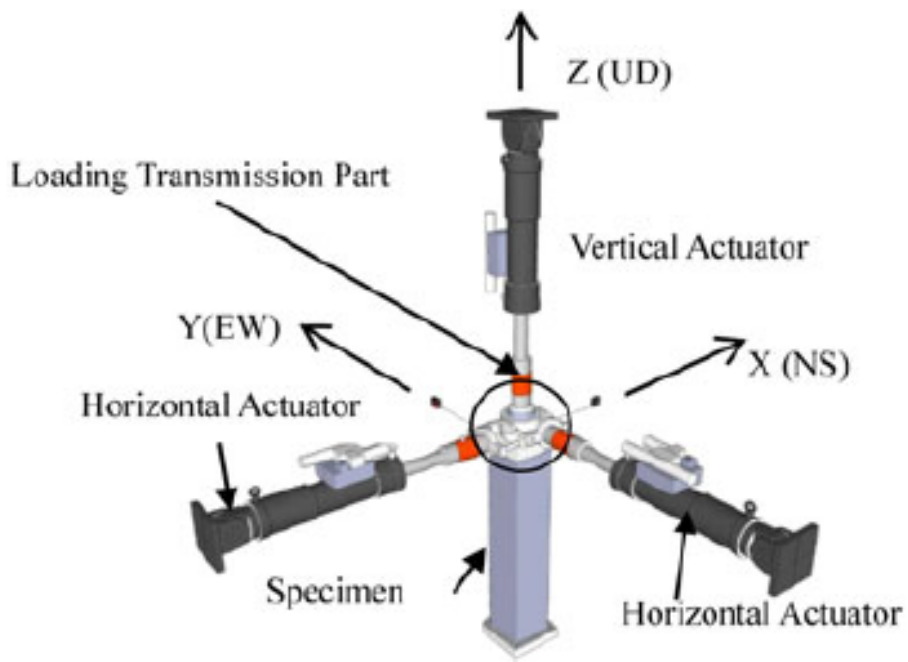

Figure 16. Loading system for 3-D PsD tests [183]

Obata and Goto [183] performed one of the earliest multi-axial PsD tests in order to investigate the effect of multi-directional loading on the ultimate limit-state behaviour of a steel bridge pier. Another example of multi-directional loading was Dang and Aoki, [184] who performed three-dimensional PsD tests of a quarter-scale stiffened square cross-section steel bridge pier subjected to vertical and bi-directional horizontal loading as shown in Figure 16. The test program provided insight into bearing capacity decrease and displacement response variations of the bridge under bi-directional loading.

Abbiati et al., [87] performed scaled (1:2.5) laboratory based PsD tests of an existing 1950's reinforced concrete bridge that was to be retrofitted using isolation devices. Hybrid numerical simulations were used to analyse the existing and retrofitted structure to aid design of the PsD experiments. Two of the twelve bridge piers formed the PS whilst the remaining ten bridge piers and deck formed the NS. The PsD tests formed part of SERIES (Seismic Engineering Research Infrastructures for European Synergies) funded project called RETRO.

\subsection{Applications of Real-time Hybrid Testing}

Igarashi et al., [37] performed a full-scale substructured RTHSTT of an idealised two DOF structural system with a tuned mass damper (TMD) providing structural control. The TMD formed the PS whilst the structural system formed the NS. Results showed that the control method using the TMD is feasible as long as the stability conditions of the test specimen and test parameters are satisfied. A similar substructured RTHT was performed by [36] of an active mass damper (AMD). 


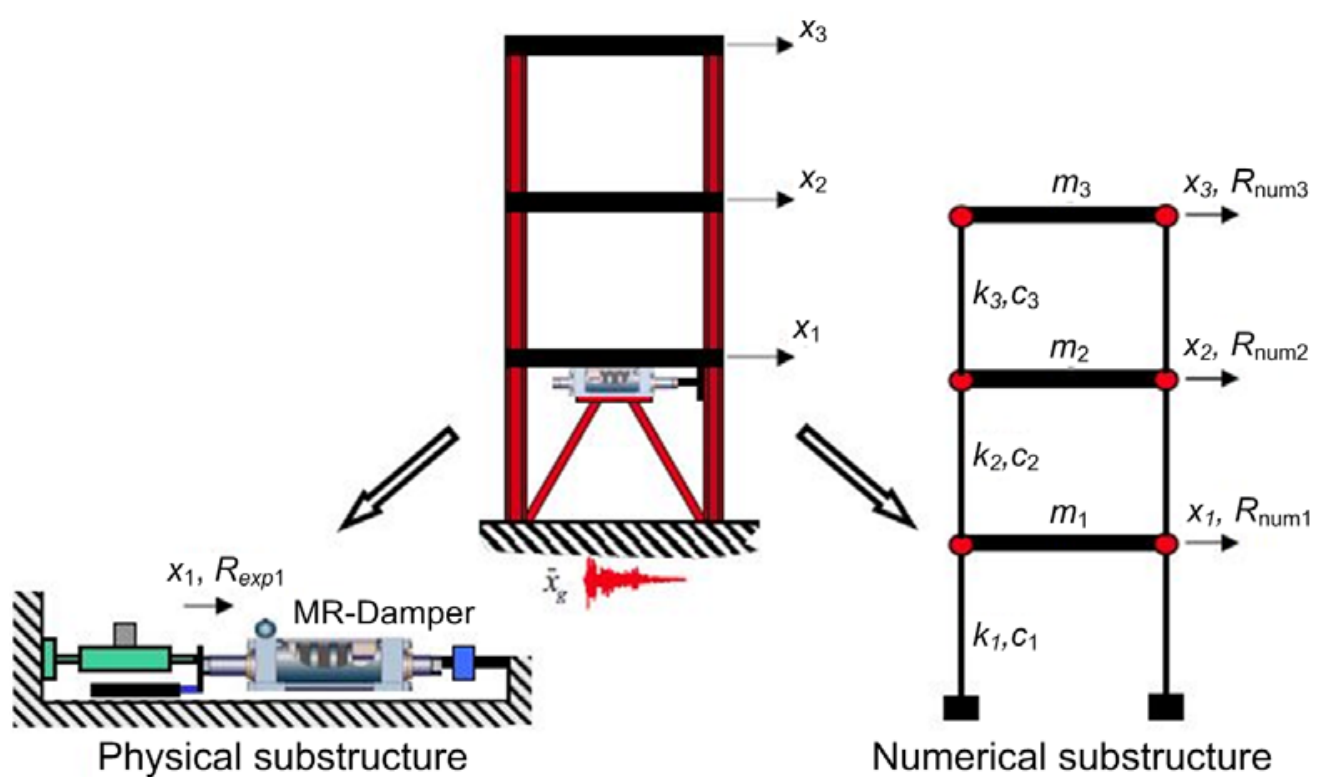

Figure 17. Schematic of real-time hybrid test of semi-active control device [2]

Carrion and Spencer, [2] described a full-scale substructured RTHT of an MR damper for semi-active control of a three-storey steel framed structure. The MR damper formed the PS whilst the remainder of the structure formed the NS (see Figure 17). Model-based feedforward compensation accounted for the variations on the actuator dynamics. The RTHT demonstrated the successful performance of the structural control algorithm. A number of other authors have also performed substructured RTHTs to investigate semi-active control of building structures using MR dampers, such as [104], [185] \& [186].

Lee et al., [43] evaluated the vibration control effect of a scaled tuned liquid damper (TLD) for a building structure using the RTHSTT method. The TLD formed the PS and a numerical structural model of a single- and three-storey steel frame formed the NSs. Feedback from the shear force signal measured by a shear type load cell located between the shake table and TLD was used in the control loop as an interaction force between the TLD and NS as shown in Figure 18. Comparison between the RHSTT method and a conventional shake table test showed good agreement. The test results showed that the TLD could effectively mitigate the seismic response of the structure investigated. 


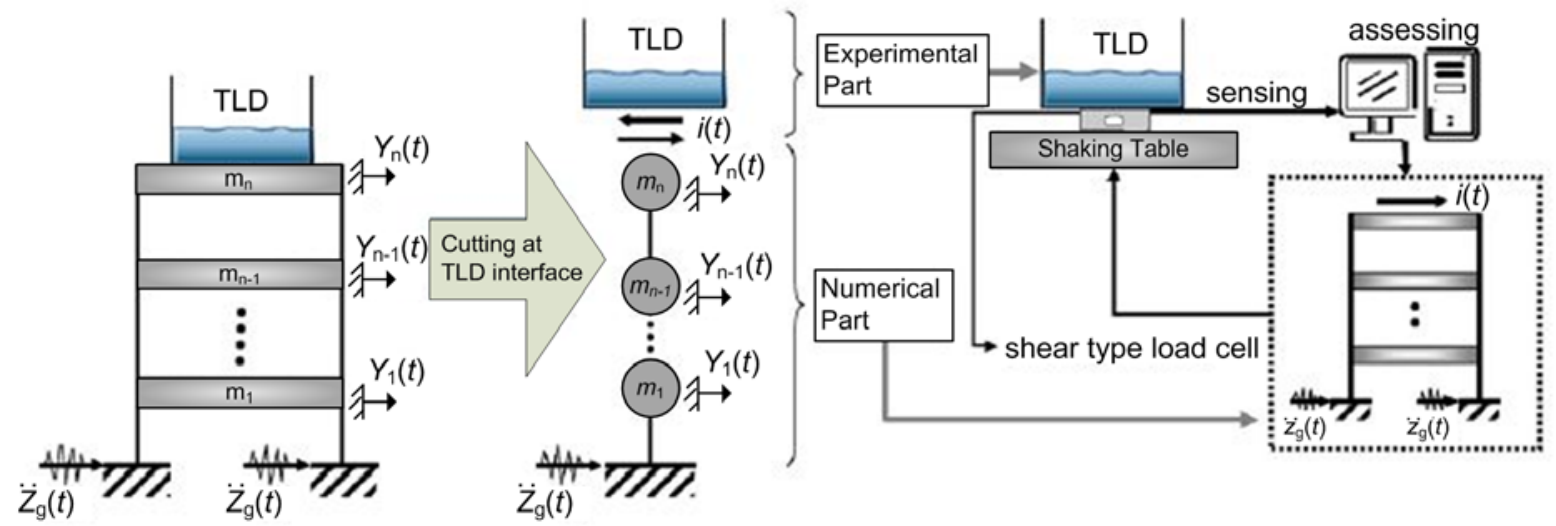

Figure 18. Conceptual view of real-time hybrid shake table test of TLD [43]

Stavridis and Shing, [187] performed a series of RTHTs of a $1 / 3$ scale three-storey suspended zipper steel frame. The bottom-storey formed the PS whilst the remainder of the structure formed the NS. The zipper struts were designed to transfer unbalanced forces up to the story above when the V-bracing in the storey below buckles. Results showed the top storey remained elastic and prevented collapse as designed.

Karavasilis et al., [188] evaluated the seismic performance of a full-scale two-storey four-bay steel moment resisting frame (MRF) structure with compressed elastomer dampers using a substructured RTHT. The tests were conducted to verify the performance-based seismic design of the structure. The experimental substructure consisted of two individual compressed elastomer dampers and the MRF formed the NS. Results showed that the steel MRF with elastomer dampers performed better than conventional special MRFs.

\section{CONCLUSIONS}

This paper presented an overview of hybrid testing and provided an introduction to the basic concepts and developments within the method. The technical developments in the test method from the mid-1970's to present such as continuous hybrid testing and the substructuring technique were presented. The paper presented an extensive overview of: numerical time integration techniques, experimental error compensation, geographically distributed hybrid testing and the application of pseudo dynamic and real-time hybrid testing methods for evaluating the seismic response of engineering structures.

The acceleration in recent years in the use of hybrid testing purely as a dynamic testing technique can be seen indicates that the pseudo dynamic and real-time hybrid test methods have matured to a point where they are now widely accepted as reliable research tools. Nevertheless, their implementation still requires quite a high level of expertise, and 
careful decisions regarding issues such as: choice of numerical integration scheme; choice of feedback variables, optimisation of actuator performance and delay compensation.

The effective force test method and distributed hybrid test method still require further technical development and validation in order to reach maturity. However, over the past 30 years, the pseudo dynamic and real-time hybrid testing methods have become robust, accurate and reliable dynamic test methods that can be used to perform seismic tests of fullscale engineering structures in a cost effective manner. In the future, distributed hybrid testing has the potential to develop into a reliable and useable research tool, with multiple substructures either within a single laboratory or more widely geographically distributed. A significant amount of research and development has been performed in hybrid testing, and as a result the method has the potential to be more widely adopted by structural engineering industry in a similar way as other industries, such as aeronautical and automotive.

\section{References}

[1] Williams, MS and Blakeborough, A. Laboratory testing of structures under dynamic loads: an introductory review. Phil. Trans. A, The Royal Society 2001, 359: 16511670.

[2] Carrion JE and Spencer FB, Jr., Model-based strategies for real-time hybrid testing, in NSEL Report Series. 2007, University of Illinois at Urbana-Champaign.

[3] Hakuno M, Shidawara M and Hara T, Dynamic destructive test of a cantilever beam controlled by an analog-computer. Trans. Jpn. Soc. Civ. Engrs., 1969(171): p. 1-9.

[4] Takanashi K, Udagawa K, Seki M, Okada T and Tanaka H, Non-linear earthquake response analysis of structures by a computer-actuator on-line system (part 1 detail of the system). Transcript of the Architectural Institute of Japan, 1975(No. 229).

[5] Bonnet PA, The development of multi-axis real-time substructure testing, in $\mathrm{PhD}$ thesis, Department of Engineering Science. 2006, University of Oxford.

[6] Mahin SA, Shing P-SB, Thewalt CR and Hanson RD, Pseudodynamic test method: current status and future directions. Journal of Structural Engineering, ASCE, 1989. 115(8): p. 2113-2128.

[7] Shing PB and Mahin SA, Computational aspects of a seismic performance test method using on-line computer control. Earthquake Engineering \& Structural Dynamics, 1985. 13(4): p. 507-526.

[8] Shing PB and Mahin SA, Cumulative experimental errors in pseudodynamic tests. Earthquake Engineering \& Structural Dynamics, 1987. 15(4): p. 409-424.

[9] Takanashi $\mathrm{K}$ and Nakashima M, Japanese activities on on-line testing. Journal of Engineering Mechanics, 1987. 113(7): p. 1014-1032.

[10] Magonette G, Development and application of large-scale continuous pseudodynamic testing techniques. Phil. Trans. R. Soc. Lond, 2001. 359: p. 1771-1799.

[11] Zhang Y, Sause R, Ricles JM and Naito CJ, Modified predictor-corrector numerical scheme for real-time pseudo dynamic tests using state-space formulation. Earthquake Engineering \& Structural Dynamics, 2005. 34(3): p. 271-288. 
[12] Takahashi, Y and Fenves, GL. Software framework for distributed experimentalcomputational simulation of structural systems. Earthquake Engineering \& Structural Dynamics, 2006, 35: 267-291.

[13] Nakashima, M, Akazawa, T and Sakaguchi, O. Integration method capable of controlling experimental error growth in substructure pseudo dynamic test. AIJ Journal of Struct. Constr. Engng., 454: 61-71.

[14] Bonelli, A, Bursi, OS, He, L, Magonette, G and Pegon, P. Convergence analysis of a parallel interfield method of heterogeneous simulations with substructuring. International Journal for Numerical Methods in Engineering, 2008, 75(7): 800-825.

[15] Dermitzakis SN and Mahin SA, Development of substructuring techniques for on-line computer controlled seismic performance testing, in Report UCB/EERC - 85/04. 1985, Earthquake Engineering Research Center, University of California, Berkeley.

[16] Nakashima $M$, Kaminoso $T$, Ishida $M$ and Ando $K$, Integration techniques for substructuring pseudodynamic test, in Proceedings 4th US National Conference On Earthquake Engineering. 1990: Palm Springs, CA, USA. p. 515-524.

[17] Vannan MT, The pseudodynamic test method with substructuring application, in $\mathrm{PhD}$ thesis, College of Engineering and Applied Sciences. 1991, University of Calorado: Boulder, Colorado.

[18] Buchet P and Pegon P, PSD testing with substructuring: implementation and use. JRC Special Publication No. I.94.25, Ispra, Italy, 1994: p. 21 pages.

[19] Pegon $P$ and Pinto AV, Pseudo-dynamic testing with substructuring at the ELSA Laboratory. Earthquake Engineering \& Structural Dynamics, 2000. 29(7): p. 905-925.

[20] Molina FJ, et al., Pseudodynamic tests on rubber base isolators with numerical substructuring of the superstructure and strain-rate effect compensation. Earthquake Engineering \& Structural Dynamics, 2002. 31(8): p. 1563-1582.

[21] Mutsuyoshi H, Machida A, Tanzo W and Mashiko N, Inelastic seismic response behaviour of RC bridge pier using pseudo-dynamic test method. Transactions of the Japan Concrete Institute, 1994. 16: p. 265-272.

[22] Molina FJ, Verzeletti G, Magonette G, Buchet P and Géradin M, Bi-directional pseudodynamic test of a full-size three-storey building. Earthquake Engineering \& Structural Dynamics, 1999. 28(12): p. 1541-1566.

[23] Geradin M and Molina FJ, Bi-Directional Pseudodynamic Test of a Full-Size Three Storey Steel Concrete Building with Rigid and Semirigid Joints, in The Paramount Role of Joints into the Reliable Response of Structures. NATO Science Series Volume 4. 2000. p. 237-256

[24] Iwata S, Iemura H, Honda A, Sakai K, Fukushima T and Sugiyama K. Hybrid earthquake loading test (Pseudo-dynamic test) of bi-directional base isolation bearing for a large pedestrian bridge. in Proceedings of the 12th World Conference on Earthquake Engineering. 2000. New Zealand.

[25] Nagata S, Kawashima K and Watanabe G, Effect of P- $\Delta$ action of actuators in a hybrid loading test, in Proceedings of 13th World Conference on Earthquake Engineering. 2004: Vancouver, Canada.

[26] Dhakal RP, Mander JB and Mashiko N, Bidirectional pseudodynamic tests of bridge piers designed to different standards. Journal of Bridge Engineering, 2007. 12(3): p. 284-295.

[27] Obata, M and Goto, Y, Development of Multidirectional Structural Testing System Applicable to Pseudodynamic Test. Journal of Structural Engineering, 2007. 133(5): p. 638-645. 
[28] Mercan O, Ricles JM, Sause R and Marullo T, Kinematic transformations for planar multi-directional pseudodynamic testing. Earthquake Engineering \& Structural Dynamics, 2009. 38(9): p. 1093-1119.

[29] Nakashima M, Kato H and Takaoka E, Development of real-time pseudo dynamic testing. Earthquake Engineering \& Structural Dynamics, 1992. 21(1): p. 79-92.

[30] Nakashima M and Masaoka N, Real-time on-line test for MDOF systems. Earthquake Engineering \& Structural Dynamics, 1999. 28(4): p. 393-420.

[31] Darby AP, Blakeborough A and Williams MS, Real-time substructure tests using hydraulic actuator. Journal of Engineering Mechanics, 1999. 125(10): p. 1133-1139.

[32] Horiuchi T, Inoue M, Konno T and Namita Y, Real-time hybrid experimental system with actuator delay compensation and its application to a piping system with energy absorber. Earthquake Engineering \& Structural Dynamics, 1999. 28(10): p. 11211141.

[33] Darby AP, Blakeborough A and Williams MS, Improved control algorithm for realtime substructure testing. Earthquake Engineering \& Structural Dynamics, 2001. 30: p. 431-448.

[34] Blakeborough A, Williams MS and Darby AP, The development of real-time substructure testing. Philosophical Transactions of The Royal Society 2001. 359: p. 1869-1891.

[35] Shing PB, Wei Z, Jung $R$ and Stauffer E. NEES fast hybrid test system at the University of Colorado. in Proceedings of the 13th World Conference in Earthquake Engineering. 2004. Vancouver, Canada.

[36] Iemura H, Igarashai A and Takahashi Y. Substructured hybrid techniques for actuator loading and shake table tests. in First Inetrnational Conference on Advances in Structural Engineering and Mechanics. 1999. Seoul, South Korea.

[37] Igarashi A, Iemura $\mathrm{H}$ and Suwa $\mathrm{T}$, Development of substructured shaking table test method, in Proceedings of the 12th World Conference on Earthquake Engineering. 2000.

[38] Horiuchi T, Inoue $\mathrm{M}$ and Konno T. Development of a real-time hybrid experimental system using a shaking table. in 12th World Conference on Earthquake Engineering. 2000. Auckland, New Zealand.

[39] Igarashi A, Iemura H, Tanaka $\mathrm{H}$ and Tsuruta D. Experimental simulation of coupled response of structural systems using the substructured hybrid shake table test method. in 13th World Conference on Earthquake Engineering. 2004. Vancouver, Canada.

[40] Neild SA, Stoten DP, Drury D and Wagg DJ, Control issues relating to real-time substructuring experiments using a shaking table. Earthquake Engineering \& Structural Dynamics, 2005. 34(9): p. 1171-1192.

[41] Shao X, Reinhorn AM and Sivaselvan MV, Real-time hybrid simulation using shake tables and dynamic actuators. Journal of Structural Engineering, ASCE, 2011. 137(7): p. 748-760.

[42] Reinhorn AM, Bruneau M, Chu SY, Shao X and Pitman MC. Large Scale Real Time Dynamic Hybrid Testing Technique- Shake Tables Substructure Testing. in Proceedings of ASCE Structures Congress. 2003. Seattle, WA.

[43] Lee S-K, Park EC, Min K-W, Lee S-H, Chung L and Park J-H, Real-time hybrid shaking table testing method for the performance evaluation of a tuned liquid damper controlling seismic response of building structures. Journal of Sound and Vibration, 2007. 302(3): p. 596-612.

[44] Dimig J, Shield C, French C, Bailey F and Clark A, Effective force testing: A method of seismic simulation for structural testing. Journal of Structural Engineering, ASCE, 1999. 125(9): p. 1028-1037. 
[45] Shield C, French CW and Timm J, Development and implementation of the effective force testing method for seismic simulation of large-scale structures. Philosophical Transaction of the Royal Society: Theme Issue on Dynamic Testing of Structures, 2001. A359: p. 1911-1929.

[46] Zhao J, French CW, Shield C and Posbergh T, Comparison of tests of a nonlinear structure using a shake table and the EFT method. Journal of Structural Engineering, ASCE, 2006. 132(9): p. 1473-1481.

[47] Thewalt CR and Mahin SA, Hybrid solution techniques for generalized pseudodynamic testing, in UCB/EERC-87/09. 1987, Earthquake Engineering Research Center, University of California, Berekeley.

[48] Pan, P, Tomofuji, H, Wang, T, Nakashima, M, Ohsaki, M and Mosalam, KM. Development of peer-to-peer (p2p) internet online hybrid test system. Earthquake Engineering \& Structural Dynamics, 2006, 35; 867-890

[49] Sivaselvan MV, Reinhorn AM, Shao X and Weinreber S, Dynamic force control with hydraulic actuators using added compliance and displacement compensation. Earthquake Engineering \& Structural Dynamics, 2008. 37: p. 1785-1800.

[50] Nakata N and Krug E, Multi-degrees-of-freedom effective force testing: a feasibility study and robust stability assessment. Earthquake Engineering \& Structural Dynamics, 2013: p. (published online).

[51] Bonelli A and Bursi OS, Predictor-corrector procedures for pseudo-dynamic tests. International Journal for Computer-aided Engineering and Software, 2005. 22(7): p. 783-834.

[52] Bonnet PA, Williams MS and Blakeborough A, Evaluation of numerical timeintegration schemes for real-time hybrid testing. Earthquake Engineering \& Structural Dynamics, 2008. 37(13): p. 1467-1490.

[53] Shing PB, Nakashima M and Bursi OS, Application of pseudodynamic test method to structural research. Earthquake Spectra, 1996. 12(1): p. 29-56.

[54] Algaard W, Integrated implementation system for pseudodynamic testing, in PhD thesis, Civil Engineering. 2001, University of Glasgow, UK.

[55] Zhao J, French C, Shield C and Posbergh T, Considerations for the development of real-time dynamic testing using servo-hydraulic actuation. Earthquake Engineering \& Structural Dynamics, 2003. 32(11): p. 1773-1794.

[56] Wu B, Bao H, Ou J and Tian S, Stability and accuracy analysis of the central difference method for real-time substructure testing. Earthquake Engineering \& Structural Dynamics, 2005. 34(7): p. 705-718.

[57] Newmark NM, A method of computation for structural dynamics. Journal of Engineering Mechanics Division, ASCE, 1959. 85(3): p. 67-94.

[58] Chang SY, Explicit pseudodynamic algorithm with unconditional stability. Journal of Engineering Mechanics, 2002. 128(9): p. 935-947.

[59] Chang SY, Improved explicit method for structural dynamics. Journal of Engineering Mechanics, 2007. 133(7): p. 748-760.

[60] Chang S, Yang Y and Hsu C, A family of explicit algorithms for general pseudodynamic testing. Earthquake Engineering and Engineering Vibration 2011. 10(1): p. 51-64.

[61] Chen C and Ricles JM, Stability analysis of SDOF real-time hybrid testing systems with explicit integration algorithms and actuator delay. Earthquake Engineering \& Structural Dynamics, 2008. 37(4): p. 597-613.

[62] Chen C and Ricles JM, Tracking error-based servo-hydraulic actuator adaptive compensation for real-time hybrid simulation. Journal of Structural Division (ASCE), 2010. 136(4): p. 432-440. 
[63] Shing PB and Manivannan $T$, On the accuracy of implicit algorithm for pseudodynamic tests. Earthquake Engineering \& Structural Dynamics, 1990. 19: p. 631-651.

[64] Hilber HM, Hughes TJR and Taylor RL, Improved numerical dissipation for time integration algorithms in structural dynamics. Earthquake Engineering \& Structural Dynamics, 1977. 5(3): p. 283-292.

[65] Bursi OS and Shing P-SB, Evaluation of some implicit time-stepping algorithms for pseudodynamic tests. Earthquake Engineering \& Structural Dynamics, 1996. 25(4): p. 333-355.

[66] Bayer V, Dorka UE and Fullerkrug U. A new algorithm for real-time sub-structure pseudo-dynamic tests. in Proceedings of the 12th World Conference on Earthquake Engineering. 2000. Auckland, New Zealand.

[67] Bursi OS, Gonzalez-Buelga A, Vulcan L, Neild SA and Wagg DJ, Novel coupling Rosenbrock-based algorithms for real-time dynamic substructure testing. Earthquake Engineering \& Structural Dynamics, 2008. 37: p. 339-360.

[68] Lamarche CP, Bonelli A, Bursi OS and Tremblay R, A Rosenbrock-W method for real-time dynamic substructuring and pseudo-dynamic testing. Earthquake Engineering \& Structural Dynamics, 2009. 38: p. 1071-1092.

[69] Bursi SO, He L, Lamarche C-P and Bonelli A, Linearly Implicit Time Integration Methods for Real-Time Dynamic Substructure Testing. Journal of Engineering Mechanics, 2010. 136(11): p. 1380-1389.

[70] Hughes TJR, Pister KS and Taylor RL, Implicit-explicit finite elements in nonlinear transient analysis. Computer Methods in Applied Mechanics and Engineering, 1979. 17-18(Part 1): p. 159-182.

[71] Combescure D and Pegon P, $\alpha$-Operator splitting time integration technique for pseudodynamic testing error propagation analysis. Soil Dynamics and Earthquake Engineering, 1997. 16(7-8): p. 427-443.

[72] Nakashima $\mathbf{M}$ and Kato $\mathrm{H}$, Experimental error growth behavior and error growth control in on-line computer test control method, in Building Research Institute. 1987, Ministry of Construction, Tsukuba, Japan.

[73] Wang Y-P, Lee C-L and Yo T-H, Modified state-space procedures for pseudodynamic testing. Earthquake Engineering \& Structural Dynamics, 2001. 30: p. 59-80.

[74] Chung J and Hulbert GM, A time integration algorithm for structural dynamics with improved numerical dissipation: the generalized-alpha method. Journal of Applied Mechanics, 1993. 60: p. 371-375.

[75] Wu B, Xu G, Wang Q and Williams MS, Operator-splitting method for real-time substructure testing. Earthquake Engineering \& Structural Dynamics, 2006. 35(3): p. 293-314.

[76] Ghaboussi J, Yun GJ and Hashash YMA, A novel predictor-corrector algorithm for sub-structure pseudo-dynamic testing. Earthquake Engineering \& Structural Dynamics, 2006. 35: p. 453-476.

[77] Hung C-C and El-Tawil S, Full operator algorithm for hybrid simulation. Earthquake Engineering \& Structural Dynamics, 2009. 38: p. 1545-1561.

[78] Hung C-C, Yen W-M and Lu W-T, An unconditionally stable algorithm with the capability of restraining the influence of actuator control errors in hybrid simulation. Engineering Structures, 2010. 42: p. 168-178.

[79] Chang SY. A time integration pseudodynamic algorithm. in Proceedings of the 11th European Conference on Earthquake Engineering. 1998. Roterdam, Holland. 
[80] Algaard W, Agar A and Bicanic N, Enhanced integral form of the Newmark time stepping scheme for pseudodynamic testing. Engineering Computations, 2001. 18(3/4): p. 676 - 689.

[81] Chen C-C and Robinson AR, Improved time-history analysis for structural dynamics. I: Treatment of rapid variation of excitation and material nonlinearity. Journal of Engineering Mechanics, 1993. 119(12): p. 2496-513.

[82] Chang S-Y, Tsai K-C and Chen K-C, Improved time integration for pseudodynamic tests. Earthquake Engineering \& Structural Dynamics, 1998. 27(7): p. 711-730.

[83] Kim SJ, Christenson RE, Wojtkiewicz SF and Johnson EA, Real-time hybrid simulation using the convolution integral method. Smart Materials and Structures, 2011. 20.

[84] Kwon O and Kammula V, Model updating method for substructure pseudo-dynamic hybrid simulation. Earthquake Engineering \& Structural Dynamics, 2013. Published online DOI: 10.1002/eqe.2307.

[85] Yang YS, Tsai KC, Elnashai AST and Hsieh T-J. An online optimization method for bridge dynamic hybrid simulations. Simulation Modelling Practice and Theory, 2012, 28:42- 54.

[86] Spencer BF, Chang C-M, Frankie TM, Kuchma DA, Silva PF and Abdelnaby AE. A phased approach to enable hybrid simulation of complex structures. Earthquake Engineering and Engineering Vibration, 2014, 13, 63-77.

[87] Abbiati G, Bursi OS, Cazzador E, Ceravolo R, Mei Z, Paolacci F and Pegon P. Pseudo-dynamic testing based on non-linear dynamic substructuring of a reinforced concrete bridge. Experimental Research in Earthquake Engineering, EU-SERIES Concluding Workshop, Series: Geotechnical, Geological and Earthquake Engineering, 2015, Vol. 35, Taucer, F., Apostolska, Roberta (Eds.), Springer.

[88] Shing PB and Mahin SA, Experimental error propagation in pseudodynamic testing, in Report UCB/EERC-83/12. 1983, Earthquake Engineering Research Center, University of California, Berkeley.

[89] Mosqueda G, Stojadinovic B and Mahin SA, Implementation and accuracy of continuous hybrid simulation with geographically distributed substructures, in UCB/EERC 2005-02. 2005, Earthquake Engineering Research Center. University of California, Berkeley.

[90] Shing PB and Mahin SA, Pseudodynamic test method for seismic performance evaluation: theory and implementation, in Report UCB/EERC-84/01. 1984, Earthquake Engineering Research Center, University of California, Berkeley.

[91] Molina FJ, Magonette G, Pegon P and Zapico B, Monitoring damping in pseudodynamic tests. Journal of Earthquake Engineering, 2011. 15(6): p. 877-900.

[92] Bonnet PA, Williams MS and Blakeborough A, Compensation of actuator dynamics in real-time hybrid tests. Journal of System Control Engineering 2006(Special Issue Paper): p. 251-264.

[93] Horiuchi T and Konno T. Development of a real-time hybrid experimental system with actuator delay compensation. in 11th World Conference on Earthquake Engineering. 1996. Acapulco, Mexico.

[94] Kobayashai H and Tamura K. Experimental study on the validity of real-time hybrid vibration experiments with a 2-dimensional and 3-degree-of-freedom model. in 12th World Conference on Earthquake Engineering. 2000. Auckland, New Zealand.

[95] Darby AP, Williams MS and Blakeborough A, Stability and delay compensation for real-time substructure testing. Journal of Engineering Mechanics, 2002. 128(12): p. 1276-1284. 
[96] Carrion JE and Spencer BF. Real-time hybrid testing using model-based delay compensation. in 4th International Conference on Earthquake Engineering. 2006. Taipei, Taiwan.

[97] Horiuchi T and Konno T, A new method for compensating actuator delay in real-time hybrid experiments. Philosophical Transaction: Mathematics, Physics and Engineering Science 2001. 359(1786): p. 1893-1909.

[98] Wallace MI, Wagg DJ and Neild SA, An adaptive polynomial based forward prediction algorithm for multi-actuator real-time dynamic substructuring. Proceedings of the Royal Society A: Mathematical, Physical and Engineering Science, 2005. 461: p. 3807-3826.

[99] Jung R-Y and Benson Shing P, Performance evaluation of a real-time pseudodynamic test system. Earthquake Engineering \& Structural Dynamics, 2006. 35(7): p. 789-810.

[100] Marshall JE, ed. Control of time-delay systems. ed. P. Peregrinus. 1979, Stevenage: New York.

[101] Shao X, Reinhorn, A. M., and Sivaselvan, M. . Real-time dynamic hybrid testing using force-based substructuring. in ASCE Structures Congress. 2006. Reston, Va.

[102] Shao X, Reinhorn AM and Sivaselvan M, Real-time hybrid simulation using shake tables and dynamic actuators. Journal of Structural Engineering, 2010. 137(7): p. 748760.

[103] Chen C, Development and numerical simulation of hybrid effective force testing method, in Dept. of Civil and Environmental Engineering. 2007, Lehigh University: Bethlehem, PA.

[104] Christenson R, Lin YZ, Emmons A and Bass B, Large-scale experimental verification of semiactive control through real-time hybrid simulation. Journal of Structural Engineering, 2008. 134(4): p. 522-534.

[105] Colgate JE, Stanley MC and Brown JM, Issues in the haptic display of tool use. IROS, 1995. 3: p. 140-145.

[106] Seible F, Hegemier GA and Igarashi A, Simulated seismic laboratory load testing of full-scale buildings. Earthquake Spectra, 1996. 12(1): p. 57-86.

[107] Wagg DJ and Stoten DP, Substructuring of dynamic systems via the adaptive minimal control synthesis algorithm. Earthquake Engineering \& Structural Dynamics, 2001. 30: p. 865-877.

[108] Lim CN, Neild SA, Stoten DP, Drury D and Taylor CA, Adaptive control strategy for dynamic substructuring tests. Journal of Engineering Mechanics, 2007. 133(864-873).

[109] Lim CN, Neild SA, Stoten DP, Taylor CA and Drury D. Using adaptive control for dynamic substructuring tests. in 13th World Conference on Earthquake Engineering. 2004. Vancouver, BC, Canada.

[110] Bonnet PA, Lim CN, Williams MS, Blakeborough A, Neild SA, Stoten DP and Taylor CA, Real-time hybrid experiments with Newmark integration, MCSmd outerloop control and multi-tasking strategies. Earthquake Engineering \& Structural Dynamics, 2007. 36(1): p. 119-141.

[111] Tu JY, Lin PY, Stoten DP and Li G, Testing of dynamically substructured, baseisolated systems using adaptive control techniques. Earthquake Engineering \& Structural Dynamics, 2010. 39: p. 661-681.

[112] Ahmadizadeh M, Mosqueda G and Reinhorn AM, Compensation of actuator delay and dynamics for real-time hybrid structural simulation. Earthquake Engineering \& Structural Dynamics, 2008. 37(1): p. 21-42.

[113] Chen C, Ricles JM and Guo T. Minimizing actuator tracking and energy errors for real-time hybrid simulation through a new adaptive compensation scheme. in 20th 
Analysis \& Computation Speciality Conference (ASCE). 2012. Chicago, Illinois, USA.

[114] Chen P-C and Tsai KC, Dual compensation strategy for real-time hybrid testing. Earthquake Engineering \& Structural Dynamics, 2013. 42: p. 1-23.

[115] Wu B, Wang Z and Bursi OS, Actuator dynamics compensation based on upper bound delay for real-time hybrid simulation. Earthquake Engineering \& Structural Dynamics, 2013((published online)).

[116] Chae Y, Kazemibidokhti K and Ricles JM, Adaptive time series compensator for delay compensation of servo-hydraulic actuator systems for real-time hybrid simulation. Earthquake Engineering \& Structural Dynamics, 2013((published online)).

[117] Kalman RE, An approach to linear filtering and prediction problems. Transactions of the ASME, Journal of Basic Engineering, 1960. 82: p. 35-45.

[118] Stengel RF, Stchastic optimal control: theory and application. 1986, New York: John Wiley \& Sons.

[119] Spencer BF, et al., NEESGRID: A distributed collaboratory for advanced earthquake engineering experiment and simulation, in 13th World Conference on Earthquake Engineering. 2004: Vancouver, BC, Canada.

[120] Takahashi Y and Fenves GL, Software framework for distributed experimentalcomputational simulation of structural systems. Earthquake Engineering \& Structural Dynamics, 2006. 35(3): p. 267-291.

[121] Campbell S and Stojadinovic B, A system for simultaneous pseudodynamic testing of multiple substructures, in Sixth U.S. National Conference on Earthquake Engineering. 1998: Seattle, Washington.

[122] Watanabe E, Sugiura K, Nagata K and Suzuka Y. Development of parallel pseudodynamic test system. in Proceedings of 10th Japan Earthquake Engineering Symposium. 1998. Yokohama, Japan.

[123] Watanabe E, Sugiura K, Nagata K, Yamaguchi T and Niwa K, Multi-phase Interaction Testing System by Means of the Internet, in 1st International Conference on Advances in Structural Engineering and Mechanics. 1999: Seoul, Korea.

[124] Watanabe E, Kitada T, Sugiura K and Nagata K, Parallel Pseudo-Dynamic Seismic Loading Test on Elevated Bridge System Through the Internet, in The Eighth East Asia-Pacific Conference on Structural Engineering and Construction. 2001: Singapore.

[125] Suzuki H, Investigation of On-Line Parallel Hybrid Experiment System using the Internet among Universities, in Department of Civil Engineering. 2002, Kyoto University: Kyoto, Japan

[126] Saleem K, Parra-Fuente J, Ojaghi M, Williams M and Blakeborough A, UK-NEES: grid services architecture for earthquake, in Proceedings of the WSEAS International Conference on Applied Computing Conference. 2008, World Scientific and Engineering Academy and Society (WSEAS): Istanbul, Turkey. p. 174-181.

[127] Yang YS, Wang SJ, Wang KJ, Tsai KC and Hsieh SH. ISEE: Internet-based simulations for earthquake engineering Part I: The database approach. in International Workshop on Steel and Concrete Composite Construction. 2003. Taipei, Taiwan.

[128] Watanabe E, Yun C, Sugiura K, Park D and Nagata K. On-line interactive testing between KAIST and Kyoto University. in Proceedings of the 14th KKNN Symposium on Civil Engineering. 2001. Kyoto, Japan.

[129] Park D-U, Yun C-B, Lee J-W, Nagata K, Watanabe E and Sugiura K, On-line pseudodynamic network testing on base-isolated bridges using Internet and wireless Internet. Experimental Mechanics 2005. 45(4): p. 331-343. 
[130] Wang KJ, Tsai KC, Wang SJ, Cheng WC and Yang YS, ISEE: Internet-based simulations for earthquake engineering Part II: The application protocol appraoch. Earthquake Engineering \& Structural Dynamics, 2007. 36: p. 2307-2323.

[131] Ma QT, Omenzetter P, Ingham JM, Butterworth JW and Pender MJ, Overview of NZNEES@Auckland, in Proceedings of 2007 New Zealand Society for Earthquake Engineers Conference. 2007: Palmerston North, New Zealand.

[132] Kim JK, KOCED Program. , in Eighth US National Conference on Earthquake Engineering (8NCEE). 2006: San Francisco, California, USA.

[133] Ojaghi M, et al., UK-NEES - Distributed Hybrid Testing Between Bristol, Cambridge and Oxford Universities: Connecting Structural Dynamics Labs to a Geotechnical Centrifuge, in Proceedings of the 9th U.S. National and 10th Canadian Conference on Earthquake Engineering. 2010: Toronto, Canada.

[134] Wang KJ, Wang SJ, Yang YS, cheng WC and Tsai KC. see: Internet-based simulations for earthquake engineering part I: The application protocol approach. in International Workshop on Steel and Concrete Composite Construction. 2003. Taipei, Taiwan.

[135] Pan P, Nakashima M and Tomofuji H, Online test using displacement-force mixed control. Earthquake Engineering \& Structural Dynamics, 2005. 34: p. 869-888.

[136] Guo Y, Xiao Y and Hu Q. NetSLab-based remote hybrid testing in current hierarchical network environment. in 4th International Conference on Earthquake Engineering. 2006. Taipei, Taiwan.

[137] Kwon O, Elnashai AS and Spencer BF, A framework for distributed analytical and hybrid simulations. Structural Engineering and Mechanics, 2008. 30(3): p. 331-350.

[138] Schellenberg A, Kim HK, Takahashi Y, Fenves GL and Mahin SA, OpenFresco. 2009, University of California, Berkeley.

[139] Yang YS, Hsieh SH, Tsai KC, Wang SJ, Wang KJ, Cheng WC and Hsu CW, ISEE: Internet-based simulations for earthquake engineering-Part I: The database approach. Earthquake Engineering \& Structural Dynamics, 2007. 36: p. 2291-2306.

[140] Yang YS, et al. Network system for a transnational collaborative pseudo-dynamic experiment of a DSCFT-pier bridge system. in 8th National Conference on Earthquake Engineering. 2006. San Francisco, CA, USA.

[141] Tada M and Kuwahara S, Basic study on the system of structural analysis by unifying various programs through internet. Journal of Structural and Construction Engineering, Transactions of the Architecture Institute of Japan, 2004. 580(6): p. 113120.

[142] Pan P, Tomofuji H, Wang T, Nakashima M, Ohsaki M and Mosalam KM, Development of peer-to-peer (P2P) internet online hybrid test system. Earthquake Engineering \& Structural Dynamics, 2006. 35(7): p. 867-890.

[143] Wang T, Mccormick J, Yoshitakae N, Pan P, Murata Y and Nakashima M. Collapse simulation of a four-storey steel moment frame by a distributed online hybrid test. Earthquake Engineering \& Structural Dynamics, 2008, 37, 955-974.

[144] Wang T, Yoshitake N, Pan P, Lee T-H and Nakashima M. Numerical characteristics of peer-to-peer (p2p) internet online hybrid test system and its application to seismic simulation of src structure. Earthquake Engineering \& Structural Dynamics, 2008, 37, 265-282.

[145] Kwon O, Nakata N, Elnashai AS and Spencer BF, A framework for multi-site distributed simulation and application to complex structural systems. Journal of Earthquake Engineering, 2005. 9(5): p. 741-753.

[146] Pearlman L, Kesselman C, Gullapalli S, Spencer BF, Futrelle J, Ricker K, Foster I, Hubbard P and Severance C, Distributed Hybrid Earthquake Engineering 
Experiments: Experiences with a Ground-Shaking Grid Application, in Proceedings of 13th IEEE Symposium on High Performance Distributed Computing. 2004: Honolulu, Hawaii, USA.

[147] Mosqueda G, Stojadinovic B, Hanley J, Sivaselvan M and Reinhorn A, Hybrid seismic response simulation on a geographically distributed bridge model. Journal of Structural Engineering, 2008. 134(4): p. 535-543.

[148] Hibbit D, Karleson B and Sorensen P, Abaqus Theory Manual Version 6.2. 2001.

[149] McKenna F, Fenves GL and Scott MH, Open system for earthquake engineering simulation. 2000, University of California, Berkeley.

[150] Vecchio FJ and Wong P, VecTor2 and FormWorks Manual. 2003, http://www.civ.utoronto.ca/vector/user_manuals/manual1.pdf.

[151] Elnashai AS, Papanikolaou V and Lee D-H, ZEUS-NL User Manual. 2002, MidAmerica Earthquake Center (MAE) Report.

[152] Gehrig D, Guide to the NEESgrid reference implementation, in Technical Report NEESgrid-2004-04. 2004.

[153] Xiao Y and Yi WJ, Proceedings of Workshop on Modern Structural Experiment (in Chinese). 2002: Hunan University, Changsha, China.

[154] Xiao Y, Hu Q, Guo YR, Zhu PS and Yi WJ. Development of a networks platform for remote hybrid dynamic testing. in Proceedings of the 13th World Conference on Earthquake Engineering. 2004. Vancouver, B.C., Canada.

[155] Xinjiang C, Tian S, Wang D and Xiao Y, Networked collaborative pseudo-dynamic testing of a multi-span bridge based on NetSLab. Earthquake Engineering and Engineering Vibration, 2009. 8(3): p. 387-397.

[156] Xu B, Lu P, Guo Y and Xiao Y. Remote structure parameter estimation on Netslab platform using responses time history. in 12th International Conference on Earth and Space 2010: Engineering, Science, Construction, and Operations in Challenging Environments (ASCE). 2010. Honolulu, Hawaii.

[157] Schellenberg A, Mahin SA and Fenves GL, Advanced implementation of hybrid simulation, in Pacific Earthquake Engineering Research Center. 2009.

[158] Mosqueda G, Stojadinovic B and Mahin SA. Geographically distributed continuous hybrid simulation. in 13th World Conference on Earthquake Engineering. 2004. Vancouver, BC, Canada.

[159] Schellenberg A, Advanced implementation of hybrid simulation, in PhD thesis, Civil Engineering. 2008, University of California, Berkeley.

[160] McCrum DP and Broderick BM, Evaluation of a substructures soft-real time hybrid test for performing seismic analysis of complex structural systems. Journal of Computers and Structures, 2013((available online)).

[161] Schellenberg A, Kim HK, Yoshikazu T and Mahin SA, OpenFresco command language manual. 2009, University of California, Berkeley.

[162] Stojadinovic B, Mosqueda G and Mahin SA, Event-driven control system for geographically distributed hybrid simulation. Journal of Structural Engineering, ASCE, 2006. 132(1): p. 68-77.

[163] Kim SJ, Christenson R and Phillips B. Geographically distributed real-time hybrid simulation of MR dampers for seismic hazard mitigation. in 20th Analysis \& Computation Specialty Conference ASCE. 2012. Chicago, Illinois.

[164] Hacker T, Eigenmann R and Rathje E, Advancing earthquake engineering research through cyberinfrastructure. Journal of Structural Engineering 2013. 139(7): p. 10991111. 
[165] Wilkins-Diehr N, Special issue: Science gateways - Common community interfaces to grid resources. Concurrency and Computation: Practice and Experience, 2007. 19(6): p. 743-749.

[166] Ojaghi M, Williams MS, Dietz MS, Blakeborough A and Lamata Martinez I. Realtime distributed hybrid testing: coupling geographically distributed scientific equipment across the internet to extend seismic testing capabilities. Earthquake Engng Struct. Dyn., 2014, 43(7), 1023-1043.

[167] Lamata Martinez I, Ioannidis I, Pegon P, Williams MS and Blakeborough A. The process and future of data integration within the European earthquake engineering laboratories. J. Computing in Civil Engng (ASCE). 2014 28(3), Paper 04014006.

[168] Lamata Martinez I, Obon Santacana F, Williams MS, Blakeborough A and Dorka UE. Celestina-Sim: a framework to support distributed testing and service integration in earthquake engineering. J. Computing in Civil Engng (ASCE). 2015 (in press)

[169] Balendra T, Lam K-Y, Liaw C-Y and Lee S-L, Behavior of Eccentrically Braced Frame by Pseudo-Dynamic Test. Journal of Structural Engineering, 1987. 113(4): p. 673-688.

[170] Shing PB, Bursi OS and Vannan MT, Pseudodynamic tests of a concentrically braced frame using substructuring tecniques. Journal of Constructional Steel Research, 1994. 29: p. 121-148.

[171] Buonopane SG and White RN, Pseusodynamic testing of masonry infilled reinforced concrete frame. Journal of Structural Engineering, 1999. 125(6): p. 578-589.

[172] Negro P, Mola E, Molina FJ and Magonette G, Full-scale PsD Testing of a Torsionally Unbalanced Three-storey Non-seismic RC Frame in Proceeding of 13th World Conference on Earthquake Engineering. 2004: Vancouver, B. C., Canada.

[173] Pinto AV, Pegon P, Magonette G and Tsionis G, Pseudo-dynamic testing of bridges using non-linear substructuring. Earthquake Engineering \& Structural Dynamics, 2004. 33(11): p. 1125-1146.

[174] Jeong S-H and Elnashai AS, Anaytical assessment of an irregular RC frame for fullscale 3D pseudo-dynamic testing part I: analytical model verification. Journal of Earthquake Engineering, 2005. 9(Issue 1 ): p. pages 95-128

[175] Jeong S-H and Elnashai AS, Analytical assessment of an irregular RC frame for fullScale 3D pseudo-dynamic testing part II: condition assessment and test deployment. Journal of Earthquake Engineering, 2005. 9(2): p. 265-284.

[176] Fardis MN and Negro P. SPEAR International Workshop. in European Commission Joint Research Centre. 2005. Ispra, Italy.

[177] McCrum DP and Broderick BM, An experimental and numerical investigation into the seismic performance of a multi-storey concentrically braced plan irregular structure. Bulletin of Earthquake Engineering, 2013. 11: p. 2363-2385.

[178] Paquette $\mathrm{J}$ and Bruneau M, Pseudo-dynamic testing of unreinforced masonry building with flexible diaphragm and comparison with existing procedures. CJournal of Construction and Building Materials, 2006. 20: p. 220-228.

[179] Tsai KC, Hsiao P-C, Wang KJ, Weng YT, Lin ML, Lin KC, Chen CH, Lai JW and Lin SL, Pseudo-dynamic tests of full-scale CFT/BRB frame - Part 1: Specimen design, experiment and analysis. Earthquake Engineering \& Structural Dynamics, 2008. 37: p. 1081-1098.

[180] Tsai K-C and Hsiao P-C, Pseudo-dynamic tests of a full-scale CFT/BRB frame - Part 2: Seismic performance of buckling-restrained braces and connections. Earthquake Engineering \& Structural Dynamics, 2008. 37(7): p. 1099-1115. 
[181] Eatherton MR, Large-scale cyclic and hybrid simulation testing and development of controlled-rocking steel building system with replacable fuses, in Civil Engineering. 2010, University of Illinois at Urbana-Champaign: Urbana, Illinois, USA.

[182] Kammula V, Erochko J, Kwon O and Christopoulos C. Performance assessment of the self centering energy dissipative (SCED) Bracing System using Hybrid Simulation. in 15th World Conference on Earthquake Engineering. 2012. Lisbon, Portugal.

[183] Obata, M. and Goto, Y., "Multi-directional pseudo dynamic experiment of steel bridge pier", Proc. Proceedings of the Third International Conference on Advances in Steel Structures, 02.Hong Kong, China. Vol. 1, pp. 171-178, 2002

[184] Dang J and Aoki T, Bidirectional loading hybrid tests of square cross-sections of steel bridge piers. Earthquake Engineering and Structural Dynamics, 2013. 42: p. 11111130.

[185] Fujitani H, Sakae H, Ito $M$ and Hiwatashi T, Response Control Performance Evaluation of MR Damper by Shaking Table Tests and Real-time Hybrid Tests. Advances in Science and Technology, 2008. 56: p. 212-217.

[186] Chae Y, Ricles J and Sause R, Large-scale experimental studies of structural control algorithms for structures with magnetorheological dampers using real-time hybrid simulation. Journal of Structural Engineering 2013. 139(7): p. 1215-1226.

[187] Stavridis A and Shing PB, Hybrid testing and modeling of a suspended zipper steel frame. Earthquake Engineering \& Structural Dynamics, 2010. 39(2): p. 187-209.

[188] Karavasilis TL, Ricles JM, Sause R and Chen C, Experimental evaluation of the seismic performance of steel MRFs with compressed elastomer dampers using largescale real-time hybrid simulation. Engineering Structures, 2011. In33(6): p. 18591869. 